\title{
Tangle analysis of difference topology experiments: Applications to a Mu protein-DNA complex
}

\author{
ISABEL K DARCY \\ JOHN LUECKE \\ MARIEL VAZQUEZ
}

\begin{abstract}
We develop topological methods for analyzing difference topology experiments involving 3-string tangles. Difference topology is a novel technique used to unveil the structure of stable protein-DNA complexes. We analyze such experiments for the Mu protein-DNA complex. We characterize the solutions to the corresponding tangle equations by certain knotted graphs. By investigating planarity conditions on these graphs we show that there is a unique biologically relevant solution. That is, we show there is a unique rational tangle solution, which is also the unique solution with small crossing number.
\end{abstract}

57M25, $92 \mathrm{C} 40$

\section{Introduction}

Bacteriophage $\mathrm{Mu}$ is a virus that infects bacteria. $\mathrm{Mu}$ transposase is involved in transposing the Mu genome within the DNA of the bacterial host. In [38], Pathania et al determined the shape of DNA bound within the Mu transposase protein complex using an experimental technique called difference topology; see Harshey and Jayaram [26; 30], Kilbride et al [31], Grainge et al [24], Pathania et al [38; 39] and Yin et al [59; 60]. Their conclusion was based on the assumption that the DNA is in a branched supercoiled form as described near the end of Section 1 (see Figures 9 and 25). We show that this restrictive assumption is not needed, and in doing so, conclude that the only biologically reasonable solution for the shape of DNA bound by Mu transposase is the one found in [38] (shown in Figure 1). We will call this 3-string tangle the PJH solution. The 3-dimensional ball represents the protein complex, and the arcs represent the bound DNA. The Mu-DNA complex modeled by this tangle is called the $\mathrm{Mu}$ transpososome.

The topological structure of the Mu transpososome will be a key ingredient in determining its more refined molecular structure and in understanding the basic mechanism of Mu transposase. See for example Mouw et al [37] and Li et al [34] where x-ray 


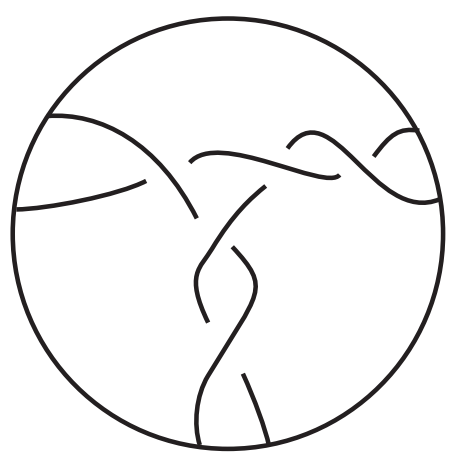

Figure 1

crystallographic data is combined with the known Tn3 resolvase topological mechanisms of binding and strand-exchange to piece together a more detailed geometrical picture of two resolvase-DNA complexes $(\operatorname{Sin}$ and $\gamma \delta)$.

In Section 1 we provide some biological background and describe eight difference topology experiments from [38]. In Section 2, we translate the biological problem of determining the shape of DNA bound by $\mathrm{Mu}$ into a mathematical model. The mathematical model consists of a system of ten 3-string tangle equations (Figure 11). Using 2-string tangle analysis, we simplify this to a system of four tangle equations (Figure 24). In Section 3 we characterize solutions to these tangle equations in terms of knotted graphs. This allows us to exhibit infinitely many different 3 -string tangle solutions. The existence of solutions different from the PJH solution raises the possibility of alternate acceptable models. In Sections 3-5, we show that all solutions to the mathematical problem other than the PJH solution are too complex to be biologically reasonable, where the complexity is measured either by the rationality or by the minimal crossing number of the 3 -string tangle solution.

In Section 3, we show that the only rational solution is the PJH solution. In particular we prove the following corollary.

Corollary 3.32 Let $\mathcal{T}$ be a solution tangle. If $\mathcal{T}$ is rational or split or if $\mathcal{T}$ has parallel strands, then $\mathcal{T}$ is the PJH solution.

In Section 4 we show that any 3 -string tangle with fewer than 8 crossings, up to free isotopy (ie allowing the ends of the tangle to move under the isotopy), must be either split or have parallel strands. Thus Corollary 3.32 implies that any solution $\mathcal{T}$ different from the PJH solution must have at least 8 crossings up to free isotopy. Fixing the framing of a solution tangle (the normal framing of Section 2) and working in 
the category of tangle equivalence - ie isotopy fixed on the boundary - we prove the following lower bound on the crossing number of exotic solutions:

Proposition 5.1 Let $\mathcal{T}$ be an in trans solution tangle. If $\mathcal{T}$ has a projection with fewer than 10 crossings, then $\mathcal{T}$ is the PJH tangle.

The framing used in [38] is different than our normal framing. With the framing of [38], Proposition 5.1 says that if Mu binds fewer than 9 crossings, then the PJH solution is the only solution fitting the experimental data. The PJH solution has 5 crossings.

We interpret Corollary 3.32 and Proposition 5.1 as saying that the PJH solution is the only biologically reasonable model for the Mu transpososome. The short length of the DNA strands (50,175 and 190 base pairs) in the transpososome and the nature of its assembly require a simple solution. Rational tangles are then a natural class in which to look for feasible solutions. They form a general class of simple tangles. Furthermore, they are freely isotopic to the trivial tangle, and one can imagine free isotopy as describing the assembly of the DNA strands within the complex. Corollary 3.32 says that the PJH solution is the unique solution in this class. In a similar vein, the shortness of the DNA strands suggest that the complex should have a projection with small crossing number. Proposition 5.1 addresses uniqueness from this point of view.

Although we describe 8 experiments from [38], we show that only 3 experiments (in cis deletion) are needed to prove the main result of Sections 3 (Corollary 3.21) and 4. A fourth experiment (in trans deletion) allows us to rule out some solutions (Section 3) and is required for the analysis in Section 5. Distinguishing which assumptions are needed for which conclusions is significant in that it allows designing the smallest number of experiments so as to minimize lab time. The remaining four experiments (inversion) were used in [38] to determine an appropriate framing for their tangle model. These four experiments are not needed in our analysis. It is interesting that, in contrast to the three in cis deletion experiments, the four inversion experiments do not determine a unique 3-string tangle among rational tangles.

The results in Sections 2-4 extend to analyzing experimental data such as Pathania et al [39] and Yin et al $[59 ; 60]$ where the products are $(2, L)$ torus links (see Hirasawa and Shimokawa [27]) or the trefoil knot (see Kronheimer et al [33]). The work in Section 3 involves the analysis of knotted graphs as in Gordon [22], Scharlemann and Thompson [45], Thompson [49], Wu [57] and Robertson et al [40]. As a by-product, we prove Theorem 3.34 and Corollary 3.37. Here if $G$ is a graph embedded in $S^{3}$, then $\operatorname{nbhd}(G)$ denotes a neighborhood of $G$ in $S^{3}$ and $X(G)=S^{3}-\operatorname{nbhd}(G)$ is the exterior of that graph. 
Theorem 3.34 Let $\widehat{G}$ be a tetrahedral graph embedded in $S^{3}$. Then $\widehat{G}$ is planar if and only if

(1) the exterior of $\widehat{G}$ has compressible boundary; and

(2) there is an edge, $\epsilon^{\prime}$, of $\widehat{G}$ such that for any edge $e \neq \epsilon^{\prime}$ of $\widehat{G}, \widehat{G}-e$ is planar.

Corollary 3.37 Suppose $\widehat{G}$ is a tetrahedral graph embedded in $S^{3}$ with the following properties:

(1) There exists three edges $e_{1}, e_{2}, e_{3}$ such that $\widehat{G}-e_{i}$ is planar.

(2) The three edges $e_{1}, e_{2}, e_{3}$ share a common vertex.

(3) There exists two additional edges, $b_{12}$ and $b_{23}$ such that $X\left(\hat{G}-b_{12}\right)$ and $X\left(\widehat{G}-b_{23}\right)$ have compressible boundary.

(4) $X(\widehat{G})$ has compressible boundary.

Then $\widehat{G}$ is planar.

In Section 3.4 we also give several examples of nonplanar tetrahedral graphs to show that none of the hypotheses in Corollary 3.37 can be eliminated.

Acknowledgments We would like to thank D Buck, R Harshey, J Mann, S Pathania, D W Sumners and C Verjovsky-Marcotte for helpful comments. We would particularly like to thank M Jayaram for many helpful discussions. We also thank M Combs for numerous figures and for typesetting help, R Scharein (SFSU) for assistance with Figure 8 using Knotplot and A Stasiak (University of Lausanne) for the electron micrograph of supercoiled DNA in Figure 9.

JL would like to thank the Institute for Advanced Study in Princeton for his support as a visiting member. This research was supported in part by the Institute for Mathematics and its Applications with funds provided by the National Science Foundation (ID and MV); by a grant from the Joint DMS/NIGMS Initiative to Support Research in the Area of Mathematical Biology (NIH GM 67242, NSF 0800285) to ID; by MBRS SCORE S06 GM052588 and Award Number P20MD000544 from the National Center On Minority Health And Health Disparities (MV).

\section{Biology background and experimental data}

DNA topology is the study of geometrical and topological properties of DNA loops and circular DNA molecules; see Bates and Maxwell [4] and Calladine et al [10]. Of particular interest are the entanglement of biopolymers and DNA protein interactions (eg Sumners [47], Bauer et al [5], Colloms et al [13], Grainge et al [24], Kilbride et al [31], Pathania et al [38] and Sumners et al [48]). 
Transposable elements, also called mobile elements, are fragments of DNA able to move along a genome by a process called transposition. Mobile elements play an important role in the shaping of a genome (see Deininger et al [18] and Sankoff [43]), and they can impact the health of an organism by introducing genetic mutations. Of special interest is that transposition is mechanistically very similar to the way certain retroviruses, including HIV, integrate into their host genome.

Bacteriophage $\mathrm{Mu}$ is a system widely used in transposition studies due to the high efficiency of Mu transposase (reviewed in Chaconas and Harshey [12]). The MuA protein performs the first steps required to transpose the Mu genome from its starting location to a new DNA location. MuA binds to specific DNA sequences which we refer to as attL and $a t t R$ sites (named after Left and Right attaching regions). A third DNA sequence called the enhancer $(\mathrm{E})$ is also required to assemble the Mu transpososome. The Mu transpososome is a very stable complex consisting of 3 segments of double-stranded DNA captured in a protein complex (see Baker and Miziuuchi [3] and Miziuuchi et al [36]). In this paper we are interested in studying the topological structure of the DNA within the Mu transpososome. This topological structure may be of use in determining a detailed molecular structure of the Mu transpososome and in understanding the basic mechanism of action of the Mu transposase on DNA.

Understanding the topology of an enzyme-DNA complex is important when composing a more detailed picture of enzymatic binding and mechanism of action, as illustrated in the following two examples. First, topological mechanisms of binding and strandexchange for the $\operatorname{Tn} 3$ resolvase were proposed in $[54 ; 46]$ : three negative supercoils are bound by the enzymes prior to recombination, and recombination happens via a $180^{\circ}$ rotation of the strands. This topological mechanism was confirmed mathematically by tangle analysis in the seminal paper by Ernst and Summers [20] (reviewed in Sumners et al [48]). The understanding of the topology of Tn3 resolvase-DNA complex has recently been used in Li et al [34] and in Mouw et al [37] in piecing together a physical model for the enzymatic binding based on the corresponding cocrystal structure for resolvases Sin by Rowland et al [41] and $\gamma \delta$, respectively. The second example deals with the site- specific recombinases XerC and XerD from Escherichia coli. The topological mechanism for XerC/XerD recombination at psi sites proposed in Colloms et al [13], was confirmed mathematically using the tangle method in Darcy [14] and Vasquez et al [50]. In [50] the results from tangle analysis were integrated with biochemical data from XerC/XerD in Alén et al [1] and Colloms et al [13], and with x-ray crystallographic data from Cre-loxP in Guo [25], to propose a more detailed molecular model for the binding of XerC/XerD enzymes to their DNA substrate. 


\subsection{Experimental design}

We base our study on the difference topology experiments of [38]. In this technique, circular DNA is first incubated with the protein(s) ${ }^{1}$ under study (eg MuA), which bind DNA. A second protein whose mechanism is well understood is added to the reaction (eg Cre). This second protein is a protein that can cut DNA and change the circular DNA topology before resealing the break(s), resulting in knotted or linked DNA. DNA crossings bound by the first protein will affect the product topology. Hence one can gain information about the DNA conformation bound by the first protein by determining the knot/link type of the DNA knots/links produced by the second protein.

In their difference topology experiments, Pathania et al [38] studied the Mu transpososome using Cre recombinase as the second protein. Cre is a site-specific recombinase from the virus Bacteriophage $\mathrm{P} 1$ which binds to recombination sites called loxP (lox stands for "locus of crossing-over"- ie, integration/deletion of DNA - and $P$ refers to P1 [28]). First, circular unknotted DNA is created containing the three binding sites for the $\mathrm{Mu}$ transpososome (attL, attR, E) and two binding sites for Cre (two loxP sites). We will refer to this unknotted DNA as substrate. The circular DNA is first incubated with the proteins required for $\mathrm{Mu}$ transposition, thus forming the transpososome complex. This complex leaves three DNA loops free outside the transpososome (Figure 2). The

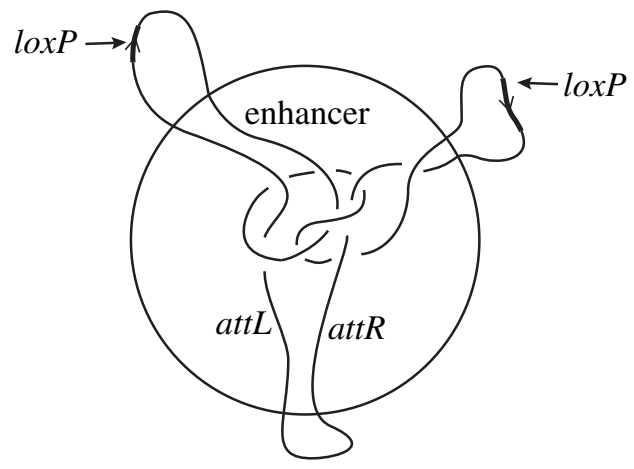

Figure 2

two loxP sites are strategically placed in two of the three outside loops. The complex is incubated with Cre enzymes, which bind the loxP sites, introduce two double-stranded

\footnotetext{
${ }^{1}$ Although we use the singular form of protein instead of the plural form, most protein-DNA complexes involve several proteins. For example formation of the $\mathrm{Mu}$ transpososome involves four MuA proteins and the protein HU. Also since these are test tube reactions and not single molecule experiments, multiple copies of the DNA-protein complex are formed by incubating many copies of the DNA substrate with a specified concentration of protein.
} 
breaks, recombine the loose ends and reseal them. A possible 2-string tangle model for the local action of Cre at these sites is shown in Figure 3 [25]. This cut-and-paste reaction may change the topology (knot/link type) of the DNA circle. Changes in the substrate's topology resulting from Cre action can reveal the structure within the $\mathrm{Mu}$ transpososome.

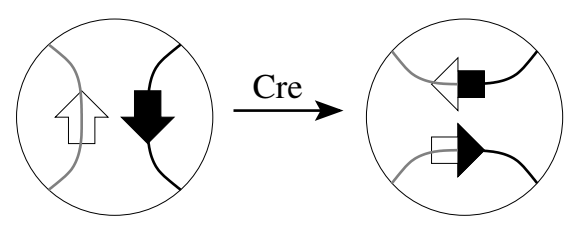

Figure 3

By looking at such topological changes, Pathania et al [38] deduced the structure of the transpososome to be that of Figure 1 (the PJH solution). In this paper we give a knot theoretic analysis that supports this deduction. We show that although there are other configurations that would lead to the same product topologies seen in the experiments, they are necessarily too complicated to be biologically reasonable.

If the orientation of both loxP sites induces the same orientation on the circular substrate (in biological terms, the sites are directly repeated), then recombination by Cre results in a link of two components and is referred to as a deletion (Figure 4, left). Otherwise the sites are inversely repeated, the product is a knot, and the recombination is called an inversion (Figure 4, right).

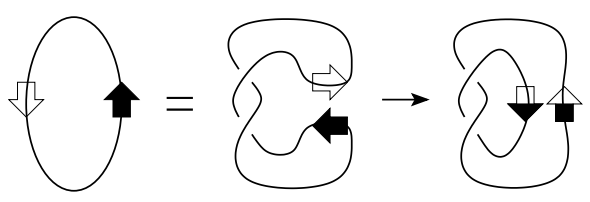

Direct repeats



Inverted repeats

Figure 4

In [38] six out of eight experiments were designed by varying the relative positions of the loxP sites and their relative orientations. The last pair of experiments involved omitting one of the Mu binding sites on the circular substrate and placing that site on a linear piece of DNA to be provided in trans as described below (Figure 5).

In the first pair of experiments from [38], loxP sites were introduced in the substrate on both sides of the enhancer sequence (E) (Figure 2). The sites were inserted with orientations to give, in separate runs, deletion and inversion products. The transpososome 
was disassembled and the knotted or linked products analyzed using gel electrophoresis and electron microscopy. The primary inversion products were $(+)$ trefoils, and the primary deletion products were 4 -crossing right-hand torus links $((2,4)$ torus links).

The assay was repeated, but now the $\operatorname{lox} P$ sites were placed on both sides of the attL sequence. The primary products were $(2,4)$ torus links for deletion, and trefoils for inversion. In a third set of experiments, the assay was repeated with the $\operatorname{lox} P$ sites on both sides of the attR sequence. The primary products were $(2,4)$ torus links for deletion, and 5-crossing knots for inversion.

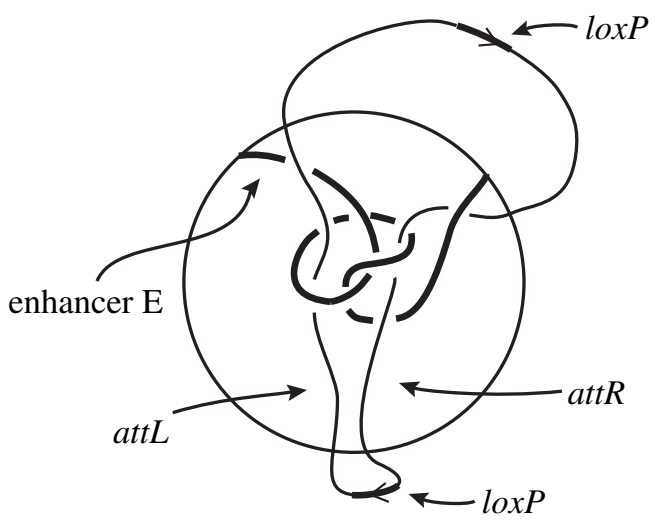

Figure 5

Recall that in these first six experiments, the three Mu binding sites, attL, attR, and the enhancer, are all placed on the same circular DNA molecule. We will refer to these six experiments as the in cis experiments to differentiate them from the final set of experiments, the in trans assay. In the in trans assay, circular DNA substrates were created that contained attL and attR sites but no enhancer site. Each loxP site was inserted between the att $L$ and $a t t R$ sites as shown in Figure 5. The enhancer sequence was placed on a linear DNA molecule. The circular substrate was incubated in solution with linear DNA molecules containing the enhancer sequence and with the proteins required for transpososome assembly. In this case, we say that the enhancer is provided in trans. The loxP sites in the resulting transpososome complex underwent Cre recombination. After the action of Cre and the disassembly of the transpososome (including the removal of the loose enhancer strand), the primary inversion products were trefoil knots, and the primary deletion products were $(2,2)$ torus links (Hopf links). 
The results of these experiments from [38] are summarized in Table $1^{2}$. Vertical columns correspond to the placement of loxP sites, eg the attL column shows inversion and deletion products when the loxP sites were placed on both sides of the att $L$ sequence.

\begin{tabular}{c|c|c|c|c} 
& enhancer & attL & attR & in trans \\
\hline Inversion & $(+)$-trefoil & trefoil & 5-crossing knot & trefoil \\
\hline Deletion & $(2,4)$ torus & $(2,4)$ torus link & $(2,4)$ torus link & $(2,2)$ torus link
\end{tabular}

Table 1

\subsection{Tangle model}

Tangle analysis is a mathematical method that models an enzymatic reaction as a system of tangle equations [20; 48]. As enzymes are often globular in shape, a protein-DNA complex is modelled as a 3-ball (the protein) with properly embedded strings (the DNA segments). 2-string tangle analysis has been successfully used to solve the topological mechanism of several site-specific recombination enzymes $[20 ; 21 ; 48 ; 24$; $14 ; 42 ; 51 ; 50 ; 7 ; 17 ; 61 ; 23 ; 15 ; 2]$. The Mu transpososome is better explained in terms of 3 -string tangles. Some efforts to classifying rational $n$-string tangles and solving $n$-string tangle equations are underway $[8 ; 9 ; 19 ; 16 ; 32]$. In this paper we find tangle solutions for the relevant 3 -string tangle equations; we characterize solutions in terms of certain knotted graphs called solution graphs and show that the PJH solution (Figure 1) is the unique rational solution.

The unknotted substrate captured by the transpososome is modeled as the union of the two 3-string tangles $\mathcal{T}_{0} \cup \mathcal{T}$, where $\mathcal{T}$ is the transpososome tangle and $\mathcal{T}_{0}$ is the tangle outside the transpososome complex. $\mathcal{T}_{0} \cup \mathcal{T}$ is represented in Figure 2. Notice that in this figure the loxP sites are placed on both sides of the enhancer sequence, but the placement of these sites varies throughout the experiments.

Figure 6 shows the action of Cre on the transpososome proposed in [38]. The experimentally observed products are indicated in this figure. For example, $E$-inversion refers to the product corresponding to inversely repeated loxP sites introduced on both sides of the enhancer sequence. However, there are other 3-string tangles, assuming the same action of Cre, that give rise to the same products. Figures 7 and 8 show two

\footnotetext{
${ }^{2}$ The chirality of the products was only determined when the loxP sites were placed on both sides of the enhancer sequence. We here assume the chirality in Table 1 , where $(2,4)$ torus link denotes the 4 -crossing right-hand torus link. If any of the products are left-hand $(2,4)$ torus links, the results of Sections 2-5 applied to these products leads to biologically unlikely solutions.
} 

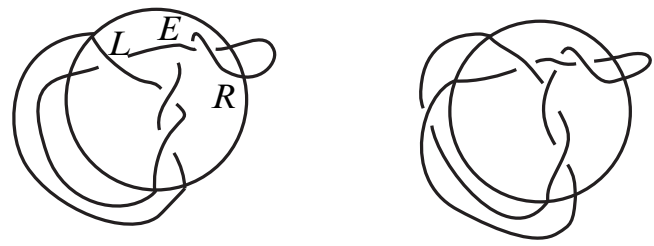

$L-$ inversion $=$ trefoil

$L$-deletion $=(2,4)$ torus
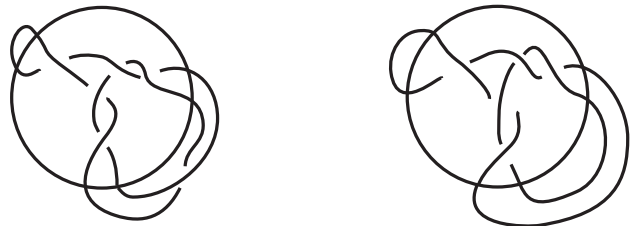

$R$-inversion $=(2,5)$ torus

$R$-deletion $=(2,4)$ torus
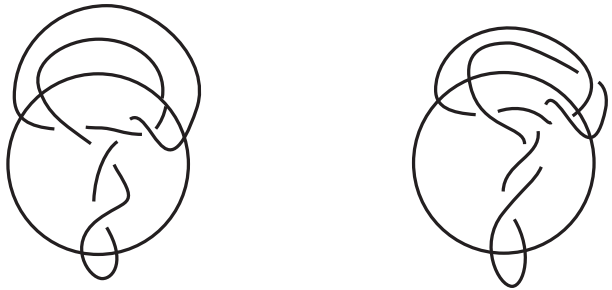

$E$-inversion $=$ trefoil

$E$-deletion $=(2,4)$ torus
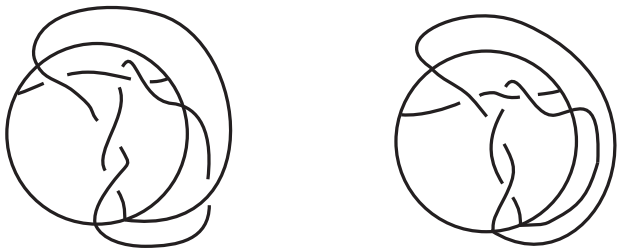

in trans inversion $=$ trefoil in trans deletion $=$ Hopf link

Figure 6

such examples. If one replaces the tangle of Figure 1 with either that of Figure 7 or 8 in Figure 6, the captions remain valid.

The loxP sites were strategically ${ }^{3}$ placed close to the $\mathrm{Mu}$ transpososome binding sites in order to prevent Cre from trapping random crossings not bound within the $\mathrm{Mu}$ transpososome. In half the experiments it was assumed that Cre trapped one extra crossing outside of the $\mathrm{Mu}$ transpososome in order to obtain the loxP sequence orientation of Figure 3 (indicated by the arrows). It was also assumed that this occurred

\footnotetext{
${ }^{3}$ Although we here describe only 8 experiments from [38], a number of experiments were performed to determine and check the effect of site placement.
} 


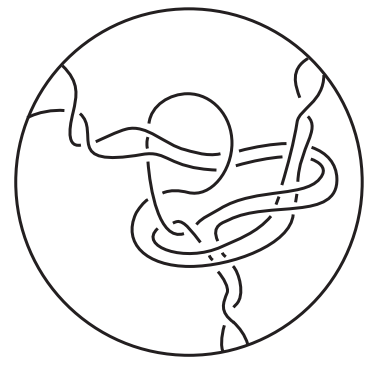

Figure 7

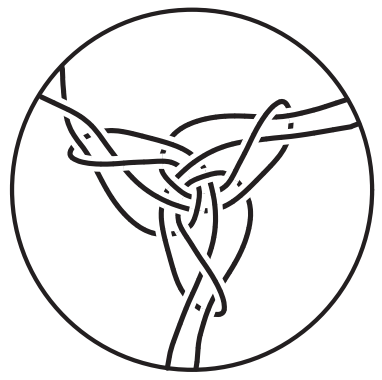

Figure 8

with the higher crossing product when comparing inversion versus deletion products. Hence a crossing outside of the Mu transpososome can be seen in Figure 6 in the case of $E$-deletion, $L$-deletion, $R$-inversion, and in trans inversion. In all other cases, it was assumed that Cre did not trap any extra crossings outside of the Mu transpososome.

The shape of DNA within the Mu transpososome was determined in [38] to be the PJH solution (Figure 1) by making a restrictive assumption regarding this DNA conformation. In their analysis, Pathania et al looked at only the most biologically likely shape: a 3 -branched supercoiled structure similar to that shown in Figure $9^{4}$. The standard form of DNA has a helical pitch of about 10.5 bp per turn of the helix [55; 53]. When the double helix is underwound or overwound, the excess torsional stress is converted into writhe, whereby the axis of the DNA double-helix crosses over itself in the form of supercoils $[4 ; 5 ; 10]$. In Figure 9, the supercoils in an unknotted, circular DNA molecule are partitioned among three branches, thus forming a branched supercoiled structure.

In terms of tangles, the assumption that the DNA is in branched supercoiled form is equivalent to asking that the three strands of bound DNA form a tangle as in Figure 25 (ie in the terminology of Section 3, that it is a standard tangle as defined in Section 3.1).

By assuming a branched supercoiled structure, [38] used their experimental results to determine the number of crossings trapped by $\mathrm{Mu}$ in each of the three branches. In Sections 2-5 we show that we are able to reach the same conclusion as [38] without assuming a branched supercoiled structure within the Mu transpososome.

\footnotetext{
${ }^{4}$ Electron microscopy of supercoiled DNA courtesy of Andrzej Stasiak, University of Lausanne.
} 

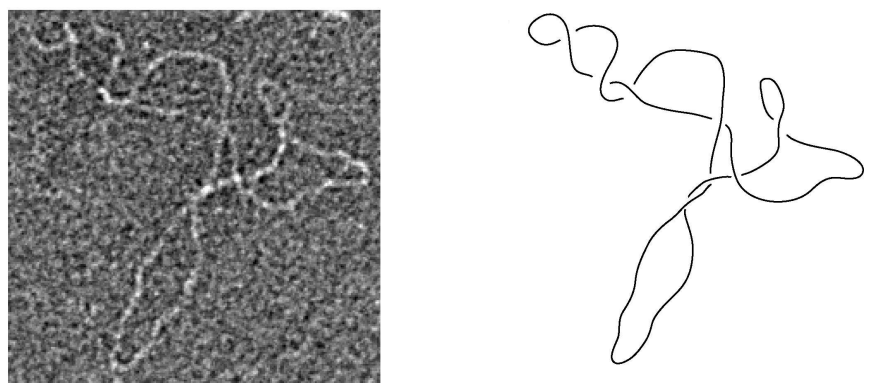

Figure 9

\section{Normal form}

\subsection{Normal form}

The substrate for Cre recombination in [38] is modeled as the 3-string tangle union $\mathcal{T} \cup \mathcal{T}_{0}$. We here introduce a framing for $\mathcal{T}$ called the normal form, which is different from that in the PJH solution (Section 1). The choice of framing affects only the arithmetic in Section 2 and does not affect any of the results in Sections 3 or 4. The results of Section 5 on the crossing number of $\mathcal{T}$ are made with respect to the normal form framing.

In Figure 10 , let $c_{1}, c_{2}, c_{3}$ be the strings of $\mathcal{T}_{0}$ and $s_{12}, s_{23}, s_{31}$ be the strings of $\mathcal{T}$. The substrate is the union of the $c_{i}$ 's and the $s_{i j}$ 's. We assume there is a projection of $\mathcal{T} \cup \mathcal{T}_{0}$ so that $c_{1}, c_{2}, c_{3}$ are isotopic (relative endpoints) onto the tangle circle and so that the endpoints of $s_{i j}$ are contiguous on the tangle circle (Figure 10). Note that

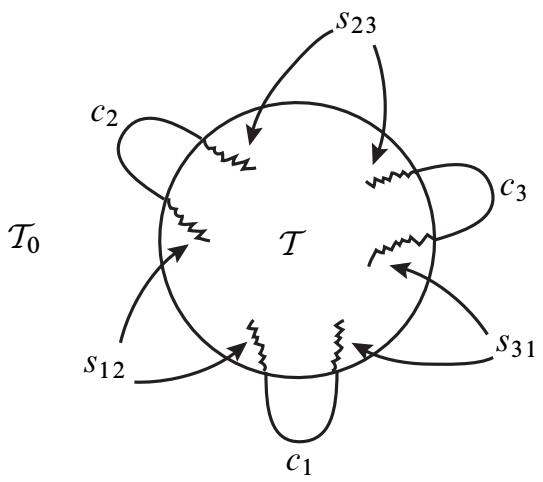

Figure 10 
this projection is different from that in [38]. It is a simple matter to convert between projections, as described below.

In each experiment the two recombination sites for Cre (loxP sites) are located on two strings $c_{i}$ and $c_{j}(i \neq j)$. Cre bound to a pair of strings $c_{i} \cup c_{j}$ can be modeled as a 2 -string tangle $P$ of type $0 / 1$. See Figure 16 where $P$ is represented by the smaller hashed 2-string tangle with two horizontal strands. Earlier studies of Cre support the assumption that Cre recombination takes $P=0 / 1$ into $R=1 / 0$, where for both tangles, the Cre binding sites are in antiparallel orientation (Figure 3) [38; 24; $25 ; 31]$. Note that from a 3 -dimensional point of view, the two sites can be regarded as parallel or antiparallel as we vary the projection $[48 ; 50 ; 52]$. With our choice of framing any Cre-DNA complex formed by bringing together two loxP sites ( eg strings $c_{i}$ and $\left.c_{j}\right)$ results in $P=(0)$ with antiparallel sites when the lox $P$ sites are directly repeated. Furthermore, it is possible that Cre recombination traps crossings outside of the $\mathrm{Mu}$ and Cre protein-DNA complexes. For mathematical convenience we will enlarge the tangle representing Cre to include these crossings which are not bound by either $\mathrm{Mu}$ or Cre but are trapped by Cre recombination. That is, the action of Cre recombination on $c_{i} \cup c_{j}$ will be modeled by taking $P=0 / 1$ into $R=1 / d$ for some integer $d$. Hence the system of tangle equations shown in Figure 11 can be used to model these experiments where the rational tangles $1 / d_{i}, 1 / v_{i}, 1 / d_{t}, 1 / v_{t}$ represent nontrivial topology trapped inside $R$ by Cre recombination, but not bound by $\mathrm{Mu}$. The tangle $\mathcal{T}$, representing the Mu transpososome (ie the Mu-DNA complex), is assumed to remain constant throughout the recombination event [48; 38]. Recall that the first six experiments where the three Mu binding sites, att $L$, attR, and the enhancer, are all placed on the same circular DNA molecule will be referred to as the in cis experiments. The remaining two experiments will be referred to as the in trans experiments since the enhancer sequence is provided in trans on a linear DNA molecule separate from the circular DNA molecule containing the att $L$ and attR sites. The tangle equation (1) in Figure 11 corresponds to the unknotted substrate equation from the first six experiments. Equations (2)-(4) correspond to three product equations modeling the three in cis deletion experiments, while Equations (5)-(7) correspond to the three product equations modeling the three in cis inversion experiments. Equation (8) corresponds to the unknotted substrate equation for the two in trans experiments, while Equations (9) and (10) correspond to the product equations modeling in trans deletion and in trans inversion, respectively. In addition to modeling experimental results in [38], these equations also model results in $[39 ; 59 ; 60]$.

Figure 10 partially defines a framing for the tangle $\mathcal{T}$. One can go further by specifying values for $d_{1}, d_{2}, d_{3}$ for the three in cis deletion experiments. We define the normal form equations to be the system of Equations (1)-(4) in Figure 11 (corresponding to the 
(1)



(8)

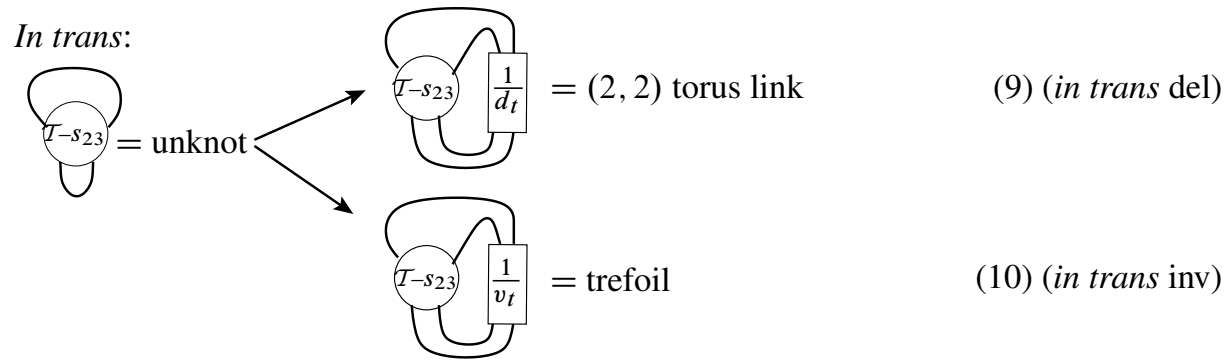

$$
\begin{gathered}
\text { where } \\
P=
\end{gathered}
$$

Figure 11

in cis deletion experiments) with the additional requirement that $d_{1}=d_{2}=d_{3}=0$. We focus first on these four equations as only the in cis deletion experiments are needed for our main results. The four inversion experiments were important experimental controls and were by [38] to determine the tangle model for the $\mathrm{Mu}$ transpososome. These four 
experiments are not needed in our analysis. It is interesting that, in contrast to the three in cis deletion experiments, the four inversion experiments do not determine a unique 3 string tangle among rational tangles.

Definition 2.1 A 3-string tangle $\mathcal{T}$ is called a solution tangle if and only if it satisfies the four normal form equations.

In the normal form equations, the action of Cre results in replacing $P=c_{i} \cup c_{j}=0 / 1$ with $R=1 / 0$ for the three in cis deletion experiments. If we wish to instead impose a framing where $P=c_{i} \cup c_{j}=0 / 1$ is replaced by $R=1 / d_{k}$ for given $d_{k}, k=1,2,3$, we can easily convert between solutions. Suppose $\mathcal{T}$ is a solution to this nonnormal form system of equations. We can move $n_{i}$ twists from $R$ into $\mathcal{T}$ at $c_{i}$, for each $i$ where $n_{i}+n_{j}=d_{k}$ (Figure 12). Hence $\mathcal{T}$ with $n_{i}$ crossings added inside $\mathcal{T}$ at $c_{i}$ for
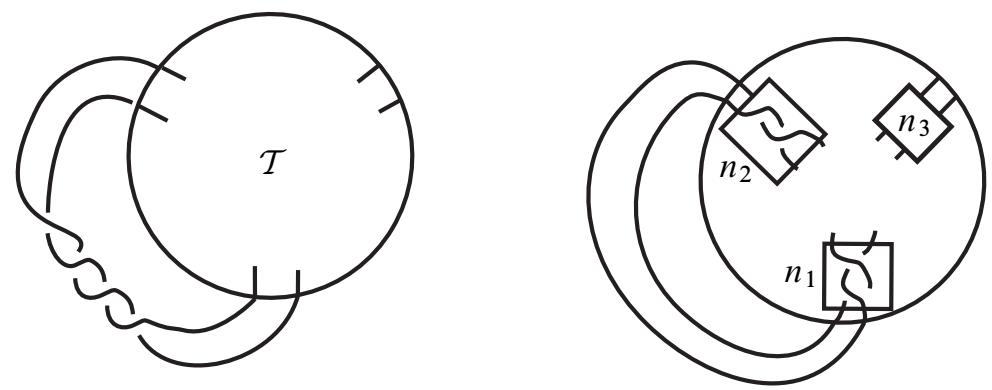

Figure 12

each $i$ is a solution tangle (for the normal form equations). Note the $n_{i}$ are uniquely determined. Similarly, if $\mathcal{T}$ is a solution tangle (for the normal form equations), then for given $d_{k}$ we can add $-n_{i}$ twists to $\mathcal{T}$ at $c_{i}$ to obtain a solution to the nonnormal form equations.

Remark For biological reasons Pathania et al chose $d_{1}=-1, d_{2}=0, d_{3}=-1$ [38]. Hence $n_{1}=0, n_{2}=-1, n_{3}=0$. This corresponds to adding a right-hand twist at $c_{2}$ to a solution in the PJH convention (such as Figure 1) to obtain a solution tangle (such as Figure 13). Conversely, by adding a single left-hand twist at $c_{2}$ to a solution tangle, we get the corresponding solution in the $\mathrm{PJH}$ convention (eg from the solution tangle of Figure 13 to the PJH solution of Figure 1).

Remark We will show that knowing the number of crossings trapped by Cre outside of the $\mathrm{Mu}$ transpososome in the three in cis deletion experiments is sufficient for determining the number of crossings trapped outside the $\mathrm{Mu}$ transpososome in all experiments. In other words choosing $d_{1}, d_{2}, d_{3}$ determines $v_{1}, v_{2}, v_{3}, v_{t}$, and $d_{t}$. 
Next we apply traditional 2-string tangle calculus [20] to the equations arising from the in cis deletion experiments.

\subsection{The three in cis deletion tangle equations}

With abuse of the $c_{i}$ notation of Figure 10, let the tangle circle be the union of arcs $c_{1} \cup x_{12} \cup c_{2} \cup x_{23} \cup c_{3} \cup x_{31}$ (Figure 13). We think of these arcs as providing a framing for the tangle $\mathcal{T}$.

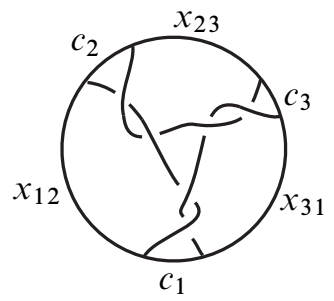

Figure 13

Let $O_{i}=\mathcal{T} \cup c_{i}$ be the 2 -string tangle obtained by capping off $s_{j i} \cup s_{i k}$ with $c_{i}$. Then $s_{k j}$ is one of the strings of the 2- string tangle $O_{i}$, let $\hat{s}_{i}$ denote the other (Figure 14)



Figure 14

By capping $\mathcal{T}$ along each $c_{i}$, we approach the problem of finding all possible 3-string tangles for the $\mathrm{Mu}$ transpososome by first solving three systems of two 2-string tangle equations (one for each $c_{i}$ ). Figure 15 illustrates the definitions of 2-string tangle addition $(+)$ and numerator closure $(N)$. Figure 16 shows a system of two 2-string tangle equations arising from $\mathrm{Cre}$ recombination on the $\mathrm{Mu}$ transpososome. $\mathcal{T} \cup c_{2}$ is represented by the 2 -string tangle which is shaded light and dark grey. The light grey portion of this 2 -string tangle represents $\mathcal{T}$. The smaller hashed 2-string tangle represents Cre bound to lox $P$ sites before (left) and after (right) recombination. Recall that the hashed $0 / 1$ tangle is referred to by $P$ (before recombination) and the hashed $1 / 0$ tangle by $R$ (after recombination). 


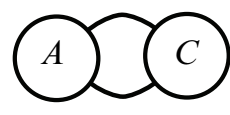

$A+C$

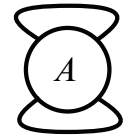

$N(A)$

Figure 15

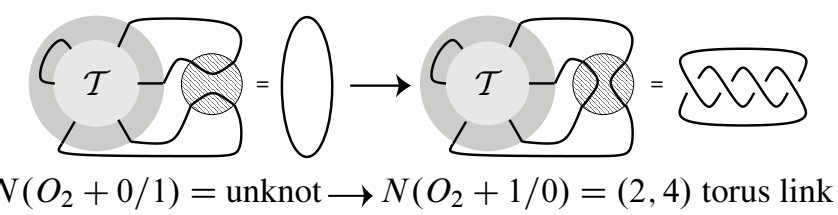

Figure 16

Lemma 2.2 (in cis deletion) Let $\mathcal{T}$ be a solution tangle. That is, consider the three in cis deletion experiments which convert unknotted substrates to right-hand $(2,4)$ torus links. Let $O_{i}=\mathcal{T} \cup c_{i}$ be the 2-string tangle obtained by capping $\mathcal{T}$ along $c_{i}$. Then $O_{i}$ is the $-1 / 4$ tangle, $i=1,2,3$.

Proof All the in cis deletion events, modeled by replacing $P=c_{j} \cup c_{k}=0 / 1$ by $R=1 / 0$ in normal form (see Figure 17), lead to identical systems of two 2-string tangle equations:

$$
\begin{aligned}
& N\left(O_{i}+0 / 1\right)=\quad \text { unknot } \\
& N\left(O_{i}+1 / 0\right)=(2,4) \text { torus link }
\end{aligned}
$$

By Hirasawa and Shimokawa [27], $O_{i}$ is a rational tangle. By tangle calculus, the system admits a unique solution $O_{i}=-1 / 4, i=1,2,3$ by Ernst and Sumners [20]

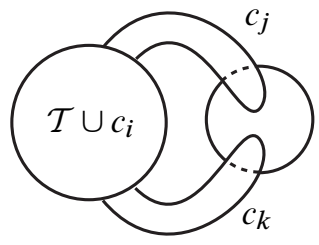

unknot

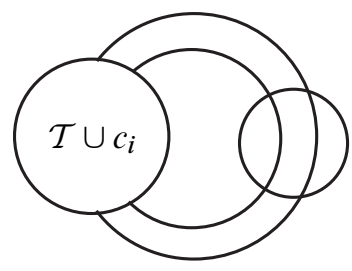

torus link

Figure 17

(see also Darcy [14] and Vasquez et al [50]). 


\subsection{The three in cis inversion tangle equations}

Each Cre inversion experiment is also modeled as a system of tangle equations:

$$
\begin{aligned}
& N\left(O_{i}+0 / 1\right)=\quad \text { unknot } \\
& N\left(O_{i}+1 / v_{i}\right)=\text { inversion product }
\end{aligned}
$$

If we assume that the DNA-protein complex is constant for each pair of deletion/inversion experiments, then by Lemma 2.2, $O_{1}=O_{2}=O_{3}=-1 / 4$. If the inversion products are known, then $v_{i}$ can be determined by tangle calculus for $i=1,2,3$. From [38] we know that both $L$-inversion and $E$-inversion produce the $(+)$-trefoil. Also $R$-inversion produces a 5 -crossing knot (see Table 1), which must be a 5 -torus knot since $O_{2}=-1 / 4$. We assume this is the $(+) 5$-torus knot, for if it were the $(-) 5$-torus knot then $v_{2}=9$ which is biologically unlikely. Hence the only biologically reasonable solutions to the inversion equations, with $O_{i}=-1 / 4, i=1,2,3$ are:

$L$-inversion, $P=0 / 1$ and $R=1 / 1$, product $=(+)$-trefoil

$R$-inversion, $P=0 / 1$ and $R=-1 / 1$, product $=(+) 5$-torus knot

$E$-inversion, $P=0 / 1$ and $R=1 / 1$, product $=(+)$-trefoil

Other possible solutions exist if $O_{i}$ is allowed to change with respect to the deletion versus inversion experiments. However, it is believed that the orientation of the Cre binding sites (inverted versus direct repeats, Figure 4) does not affect the transpososome configuration.

\subsection{Linking number considerations}

In Lemma 2.3 we compute linking numbers related to the deletion experiments. Assume $\mathcal{T}$ is a solution tangle. First, let $\hat{x}_{i}$ be the arc on the tangle circle given by $x_{j i} \cup c_{i} \cup x_{i k}$. We compute the linking number between $x_{j k} \cup s_{j k}$ and $\hat{s}_{i} \cup \hat{x}_{i}$, using the orientation induced from the tangle circle into each component and using the sign convention in Figure 18. The linking number quantifies the pairwise interlacing of arcs after capping off.

Second, we compute the linking number of $x_{i j} \cup s_{i j}$ and $x_{k i} \cup s_{k i}$, with sign and orientation conventions as before. This linking number calculation quantifies the interlacing between any two $\operatorname{arcs}$ in $\mathcal{T}$.

Lemma 2.3 Assume $\mathcal{T}$ is a solution tangle. Then

$$
\begin{aligned}
\ell k\left(x_{j k} \cup s_{j k}, \hat{s}_{i} \cup \hat{x}_{i}\right) & =-2 \\
\ell k\left(x_{i j} \cup s_{i j}, x_{k i} \cup s_{k i}\right) & =-1
\end{aligned} \quad\{i, j, k\}=\{1,2,3\} .
$$






$(+)$

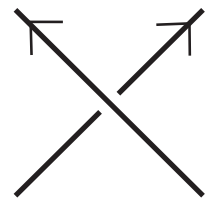

$(-)$

Figure 18

Proof The first equation follows from Lemma 2.2.

To get the second equation we note that $\widehat{s}_{i} \cup \widehat{x}_{i}$ can be obtained via a banding connecting $x_{i j} \cup s_{i j}$ with $x_{k i} \cup s_{k i}$ along $c_{i}$ as indicated in Figure 19. Then for $\{i, j, k\}=\{1,2,3\}$, $-2=\ell k\left(x_{j k} \cup s_{j k}, \hat{s}_{i} \cup \hat{x}_{i}\right)=\ell k\left(x_{j k} \cup s_{j k}, x_{i j} \cup s_{i j}\right)+\ell k\left(x_{j k} \cup s_{j k}, x_{k i} \cup s_{k i}\right)$. Solving three equations $(\{i, j, k\}=\{1,2,3\})$, we obtain $\ell k\left(x_{i j} \cup s_{i j}, x_{j k} \cup s_{j k}\right)=-1$.
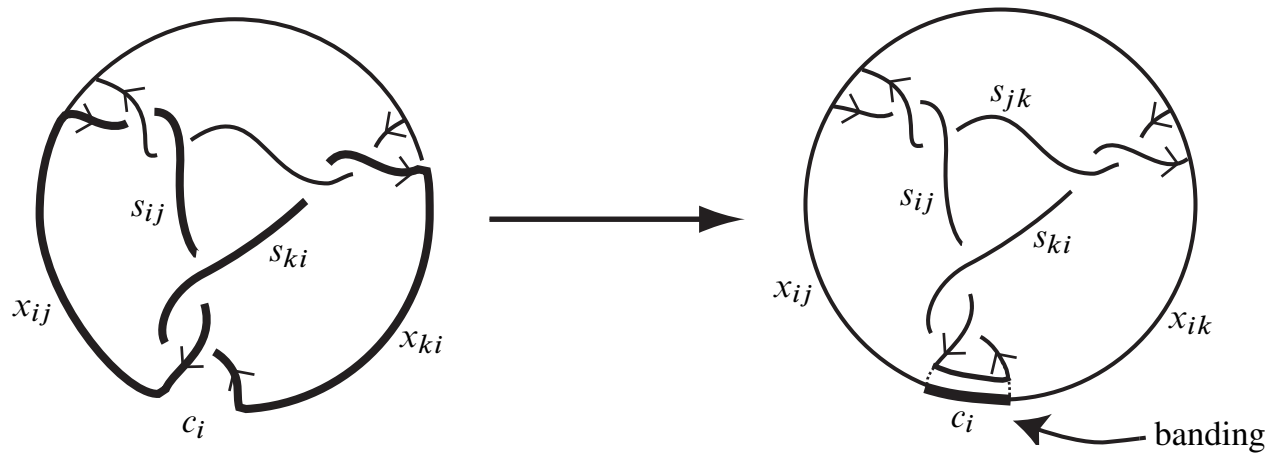

Figure 19

In the next section we will see how the in cis deletion results extend to the analysis of the remaining experiments (in trans deletion and inversion).

\subsection{In trans experiments}

In the in trans portion of [38] (described in Section 1) the enhancer sequence is not incorporated into the circular DNA substrate; it remains separate in solution on its own linear molecule when the transpososome is formed (Figure 20). Assuming the transpososome complex forms the same 3-string tangle in this context, the transpososome writes the substrate as a union of two 2 -string tangles: $\mathcal{T}_{0}^{\prime}$, a trivial tangle outside 
the transpososome; and $\mathcal{T}^{\prime}=\mathcal{T}-s_{23}$, the tangle formed by the att $R$ and att $L$ strands (where $s_{23}$ is the enhancer strand in $\mathcal{T}$ ).



Figure 20

Lemma 2.4 (in trans deletion) Let $\mathcal{T}$ be a solution tangle. Suppose $\mathcal{T}$ also satisfies equations (8) and (9) in Figure 11 where $d_{t}$ is as shown in this figure. Then $d_{t}=0,4$ and $\mathcal{T}-s_{23}=1 /-2$.

Proof When Cre acts on the loxP sites in the in trans experiment, we assume that it takes the $0 / 1$ tangle to $1 / d_{t}$ (Figure 21). The Cre deletion product in trans is a

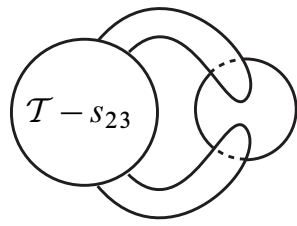

unknot



Hopf link

Figure 21

Hopf link (ie a $(2,2)$ torus link). Hence we are solving the 2 -string tangle equations, $N\left(\left(\mathcal{T}-s_{23}\right)+0 / 1\right)=$ unknot, $N\left(\left(\mathcal{T}-s_{23}\right)+1 / d_{t}\right)=(2,2)$ torus link. By [6] $\mathcal{T}-s_{23}$ is rational, and by tangle calculus [20], $\mathcal{T}-s_{23}=1 /\left( \pm 2-d_{t}\right)$, which implies that $\ell k\left(x_{31} \cup s_{31}, x_{12} \cup s_{12}\right)=\left( \pm 2-d_{t}\right) / 2$. By Lemma 2.3, $\ell k\left(x_{31} \cup s_{31}, x_{12} \cup s_{12}\right)=-1$. Hence $\left( \pm 2-d_{t}\right) / 2=-1$. Thus $d_{t}=0,4$ and $\mathcal{T}-s_{23}=1 /-2$.

Remark (In trans inversion) If $\mathcal{T}-s_{23}=1 /-2$, then the in trans Cre inversion product is $N\left(\left(\mathcal{T}-s_{23}\right)+1 / v_{t}\right)=N\left(1 /-2+1 / v_{t}\right)=\left(2,2-v_{t}\right)$ torus knot. A $(2,3)$ torus knot (ie the trefoil) in trans inversion product implies $v_{t}=-1$. 


\subsection{Summary}

The next proposition summarizes the results in this section.

Proposition 2.5 Let $\mathcal{T}$ be a solution tangle which also satisfies the in trans deletion experiments, and let $s_{23}$ correspond to the enhancer strand in the [38] experiments. Then

$$
O_{i}=\mathcal{T} \cup c_{i}=-\frac{1}{4}, \quad \mathcal{T}-s_{23}=-\frac{1}{2}
$$

The in trans deletion reaction of Cre is modeled by replacing $c_{1} \cup\left(c_{2} \cup x_{23} \cup c_{3}\right)=0 / 1$ by the $1 / 0$ or $1 / 4$ tangle (ie $d_{t}=0,4$; see Figure 23 ).

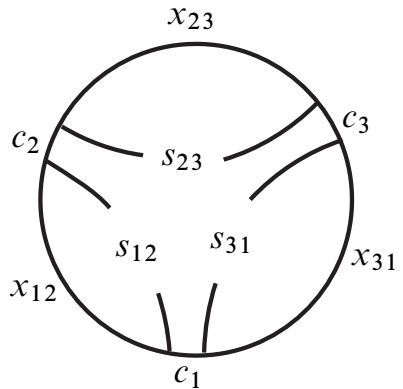

Figure 22

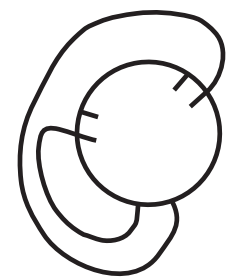

Figure 23

Remark In declaring $O_{i}=-1 / 4$ we are using the framing coordinate of the tangle circle with $c_{j} \cup c_{k}=0 / 1, x_{j k} \cup \hat{x}_{i}=1 / 0$.

Proposition 2.5 can be generalized to products of deletion that are $\left(2, L_{i}\right)$ torus links, and a $\left(2, L_{t}\right)$ torus link for the in trans experiment:

Proposition 2.6 Let $\mathcal{T}$ satisfy Equations (1)-(4), (8)-(9) of Figure 11 except that the in cis deletion experiments produce $\left(2, L_{i}\right)$ torus links, $L_{i} \neq \pm 2$, for $i=1,2,3$ and the in trans deletion experiment results in a $\left(2, L_{t}\right)$ torus link. Assume $d_{1}=d_{2}=d_{3}=0$ and that $s_{23}$ corresponds to the enhancer strand. Then

$$
O_{i}=\mathcal{T} \cup c_{i}=-\frac{1}{L_{i}}, \quad \mathcal{T}-s_{23}=\frac{1}{\left(L_{2}+L_{3}-L_{1}\right) / 2} .
$$

If $\left|L_{t}\right|=2$, then $d_{t}=\left(-L_{j}+L_{i}+L_{k} \pm 4\right) / 2$. If $\left|L_{t}\right| \neq 2$, then $d_{t}=\left(-L_{j}+L_{i}+\right.$ $\left.L_{k}-2 L_{t}\right) / 2$. 
Note similar results hold if $L_{i}= \pm 2$ for $i=1,2$, and/or 3, but as this breaks into cases, we leave it to the reader.

Remark Sections 3 and 4 rely on the fact the tangles in Figure 24 are all of the form $1 / m_{i}$. This would still be the case no matter the choice of framing (discussed at the beginning of this section) or the type of the $\left(2, L_{i}\right)$ torus link products. Hence the results of Sections 3 and 4 apply more generally.

Note that a 3 -string tangle $\mathcal{T}$ is a solution tangle if and only if it satisfies the condition $O_{i}=\mathcal{T} \cup c_{i}=-1 / 4$ for $i=1,2,3$ (see Definition 2.1 and Lemma 2.2). This corresponds to the first three equations in Figure 24. The main result in Section 3 (Corollary 3.32) only depends on the three in cis deletion experiments.



Figure 24

If in addition a solution tangle satisfies the in trans deletion equation, then we call it an in trans solution tangle:

Definition 2.7 A 3-string tangle $\mathcal{T}$ is called an in trans solution tangle if and only if it satisfies Equation (2-2) of Proposition 2.5. This is equivalent to a tangle satisfying all the equations of Figure 24. 
Note: Proposition 2.5 reduces the study of transpososome tangles to that of in trans solution tangles. In Section 3 we classify solution tangles in terms of solution graphs and use this to show that the only solution tangle (and hence the only in trans solution tangle) that is rational is the $\mathrm{PJH}$ tangle.

Remark The proof of Propositions 2.5 and 2.6 use the deletion products only. As the remaining analysis depends only on Proposition 2.5, we see that the inversion products are unnecessary for our analysis. However, the inversion products were used to determine the framing in [38].

\section{Solution graphs}

In Sections 3.1 and 3.2 we relate solution tangles to wagon wheel graphs and tetrahedral graphs. We use wagon wheel graphs to find an infinite number of solution tangles in Section 3.1. Recall by Definition 2.1 that a solution tangle is a 3 -string tangle satisfying the three in cis deletion equations (ie $O_{i}=\mathcal{T} \cup c_{i}=-1 / 4$ for $i=1,2,3$ in Equation (2-2) of Proposition 2.5, the first three equations of Figure 24), while by Definition 2.7, an in trans solution tangle is a solution tangle which also satisfies the in trans deletion equation $\mathcal{T}-s_{23}=-1 / 2$. In Section 3.3 we show that the only rational solution tangle is the PJH tangle. In Section 3.5, we extend this result to tangles which are split or have parallel strands using results in Section 3.4 regarding the exterior of a solution graph. In Section 3.6, we show that if a solution tangle is also an in trans solution tangle, then its exterior is a handlebody. We use this in Section 3.7 to investigate the planarity of tetrahedral graphs.

\subsection{Solution tangles are carried by solution graphs}

Definition 3.1 A 3-string tangle is standard if and only if it can be isotoped rel endpoints to Figure 25. A standard tangle with $n_{i}=-2$ is called the PJH tangle.
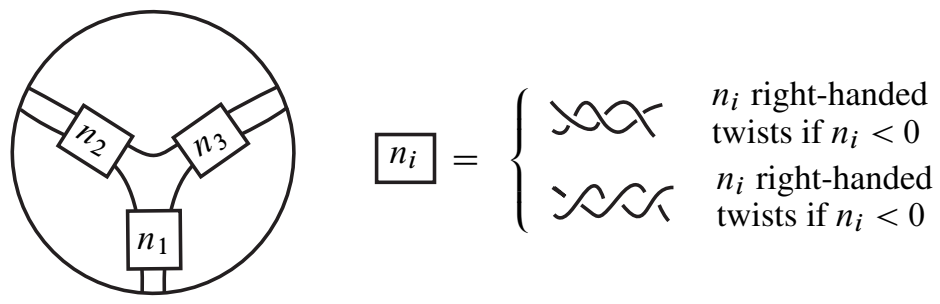

Figure 25

Note that a standard tangle is one that can be put in branched supercoiled form. 
Lemma 3.2 If a solution tangle is standard, then it is the PJH tangle.

Proof Write and solve the three equations, $n_{i}+n_{j}=-4$, describing the integral tangles resulting from capping along individual $c_{k}$.

Lemma 3.3 If an in trans solution tangle is standard, then it is the PJH tangle.

Proof By Lemma 3.2, since an in trans solution tangle is also a solution tangle.

Remark The assumption in [38] of the branched supercoiled structure (see the end of Section 1) is equivalent to assuming the in trans solution tangle is standard. In that paper, the authors deduced the transpososome configuration with a method similar to that of Lemma 3.2. The PJH solution thus obtained is equivalent to our PJH tangle when normal framing is imposed.

Definition 3.4 The abstract wagon wheel is the graph of Figure 26. The vertices are labelled $v_{1}, v_{2}, v_{3}$; the edges labelled $e_{1}, e_{2}, e_{3}, b_{12}, b_{23}, b_{31}$. A wagon wheel graph, $G$, is a proper embedding of the abstract wagon wheel into $B^{3}$ with the endpoints of the $e_{i}$ in the 10,2, and 6 o'clock positions on the tangle circle (eg Figure 27). Two wagon wheel graphs are the same if there is an isotopy of $B^{3}$, which is fixed on $\partial B^{3}$, taking one graph to the other.

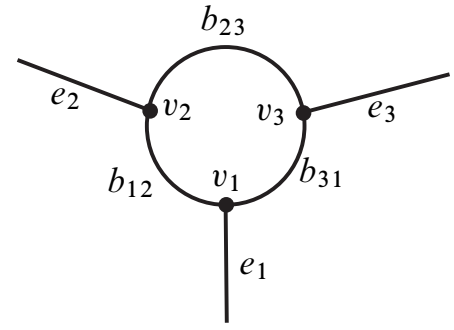

Figure 26

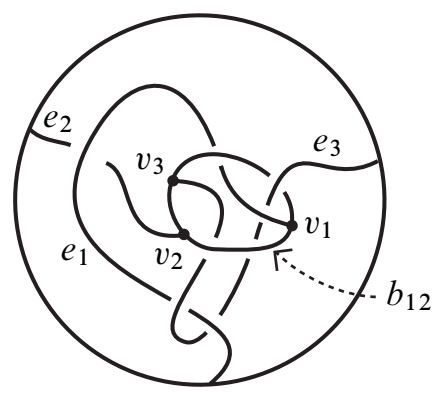

Figure 27

Definition 3.5 If a properly embedded graph lies in a properly embedded disk in the 3-ball, we call it planar.

Figure 28 gives examples of planar and nonplanar wagon wheel graphs. Note that the nonplanar graph in Figure 28 contains the knotted arc $e_{2} \cup b_{23} \cup e_{3}$. The disk in which a planar wagon wheel lies may be taken to be the one given by the plane of the page, whose boundary is the tangle circle. 

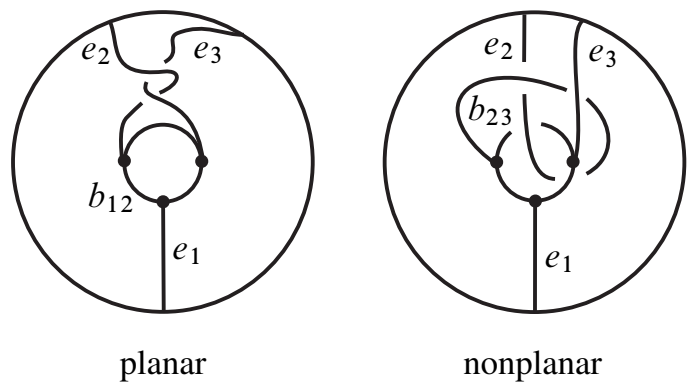

Figure 28

Definition 3.6 Let $G$ be a wagon wheel graph, and let $\operatorname{nbhd}(G)$ denote a regular neighborhood of $G$ in the 3-ball. Let $\mathcal{J}_{1}, \mathcal{J}_{2}, \mathcal{J}_{3}$ be meridian disks of $\operatorname{nbhd}(G)$ corresponding to edges $e_{1}, e_{2}, e_{3}$. Let $J_{i}=\partial \mathcal{J}_{i}$. Let $\Gamma_{12}, \Gamma_{23}, \Gamma_{31}$ be meridian disks of $\operatorname{nbhd}(G)$ corresponding to edges $b_{12}, b_{23}, b_{31}$. Let $\gamma_{i j}=\partial \Gamma_{i j}$. See Figure 29.

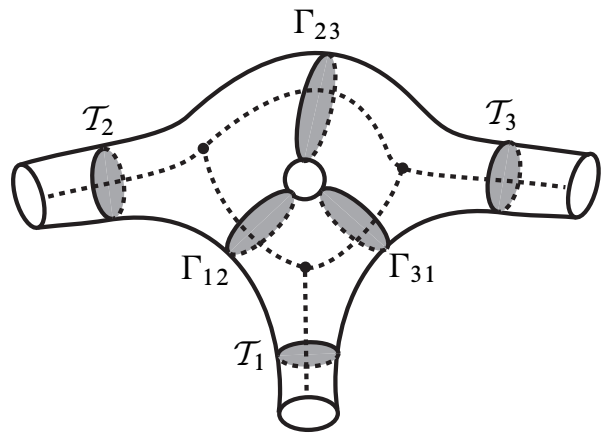

$\operatorname{nbhd}(G)$

Figure 29

Definition 3.7 $X(G)=$ exterior of $G=B^{3}-\left(\operatorname{nbhd}(G) \cup \operatorname{nbhd}\left(\partial B^{3}\right)\right)$. Note that $\partial X(G)$ is a surface of genus 3 .

Definition 3.8 $G$ is a solution graph if and only if it is a wagon wheel graph with the property that deleting any one of the edges $\left\{e_{1}, e_{2}, e_{3}\right\}$ from $G$ gives a subgraph that is planar.

Definition 3.9 $G$ is an in trans solution graph if and only if it is a wagon wheel graph with the property that deleting any one of the edges $\left\{e_{1}, e_{2}, e_{3}, b_{23}\right\}$ from $G$ gives a subgraph that is planar. 


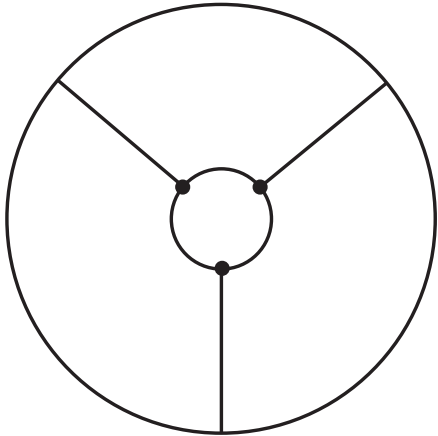

$G$

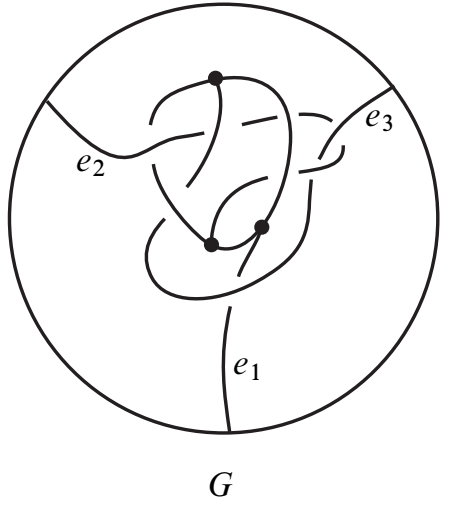

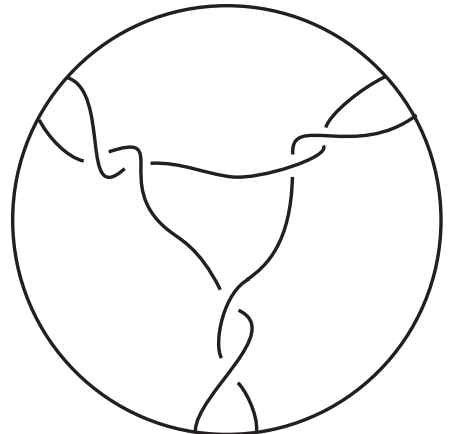

$\mathcal{T}(G)$

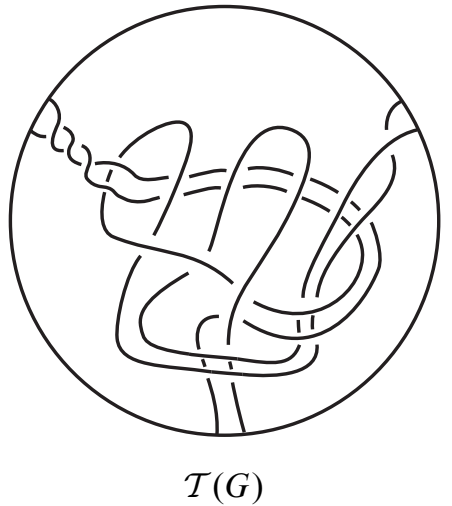

Figure 30

The graphs $\mathrm{G}$ in Figure 30 are in trans solution graphs.

Definition 3.10 Let $G$ be a wagon wheel graph. A 3 -string tangle $\mathcal{T}$ is carried by $G$, written $\mathcal{T}(G)$, if and only if its 3 -strings $s_{12}, s_{23}, s_{31}$ can be (simultaneously) isotoped to lie in $\partial \operatorname{nbhd}(G)$ such that

(1) $s_{i j}$ intersects each of $J_{i}, J_{j}$ once;

(2) $s_{i j}$ intersects $\gamma_{i j}$ once and is disjoint from the remaining $\gamma_{s t}$.

Figure 30 gives examples of tangles carried by wagon wheel graphs.

Lemma 3.11 If $\mathcal{T}_{1}, \mathcal{T}_{2}$ are both carried by $G$, then they are isotopic in $B^{3}$ (rel endpoints) up to twisting $\operatorname{nbhd}(G)$ along the $\mathcal{J}_{i}$ (ie they differ by twists at the ends).

Proof Left to the reader. 
Lemma 3.12 If a 3 -string tangle $\mathcal{T}$ is carried by a planar wagon wheel graph $G$ then $\mathcal{T}$ is standard.

Proof There is an isotopy of $B^{3}$, keeping the boundary of $B^{3}$ fixed, taking $G$ to the planar wagon wheel graph of Figure 30 (upper left). Apply Lemma 3.11 to see $\mathcal{T}$ as standard.

The following theorem and corollary let us work with graphs rather than tangles. The solution graph economically encodes the conditions needed for a solution tangle.

Theorem 3.13 Let $\mathcal{T}$ be a solution tangle, then $\mathcal{T}$ is carried by a solution graph. Conversely, a solution graph carries a unique solution tangle.

Proof Let $\mathcal{T}$ be a solution tangle with strands $s_{12}, s_{23}, s_{31}$. We show that $\mathcal{T}$ is carried by a solution graph $G$. Let $C=c_{1} \cup s_{12} \cup c_{2} \cup s_{23} \cup c_{3} \cup s_{31}$, recalling that the $c_{i}$ are the capping arcs of Figure 22. For each $i=1,2,3$, let $P_{i}$ be a point in $c_{i}$. Isotop $C$ into the interior of $B^{3}$ by pushing each $c_{i}$ slightly to the interior. Under the isotopy $P_{i}$ traces out an $\operatorname{arc} e_{i}$ from $P_{i} \in \partial B_{3}$ to $C$. Define $G=C \cup e_{1} \cup e_{2} \cup e_{3}$. See

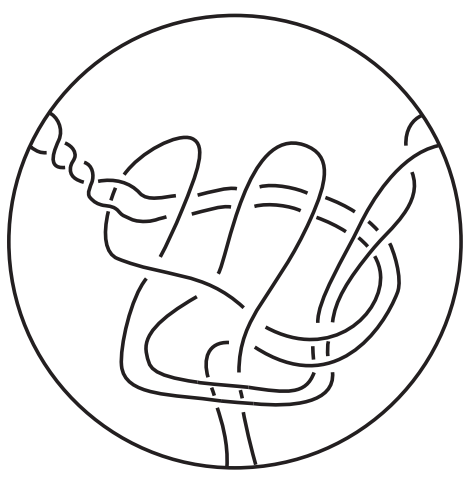

$\mathcal{T}$

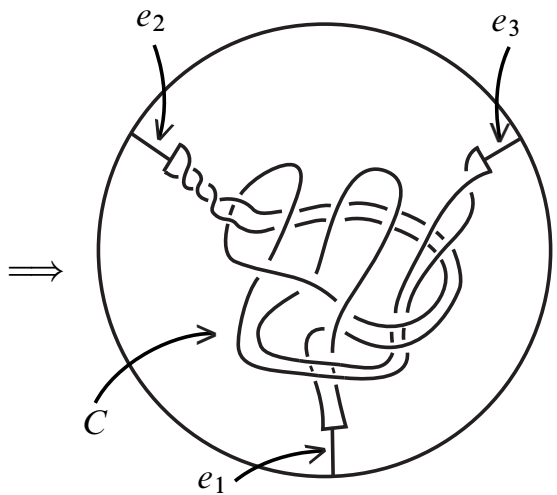

$G$

Figure 31

Figure 31. $G$ is a wagon wheel graph that carries $\mathcal{T}$. Note that the strings $s_{i j}$ of $\mathcal{T}$ correspond to the edges $b_{i j}$ of $G$. The conditions for $\mathcal{T}$ to be a solution tangle now correspond to those defining $G$ as a solution graph. For example, if $\mathcal{T}$ is a solution tangle, $s_{23} \cup\left(s_{12} \cup c_{1} \cup s_{31}\right) \cup c_{2} \cup c_{3}$ (where $c_{1}$ has been pushed into the interior of $B^{3}$ ) lies in a properly embedded disk $D$ in $B^{3}$. Hence $G-e_{1}$ lies in $D$ and is planar. 


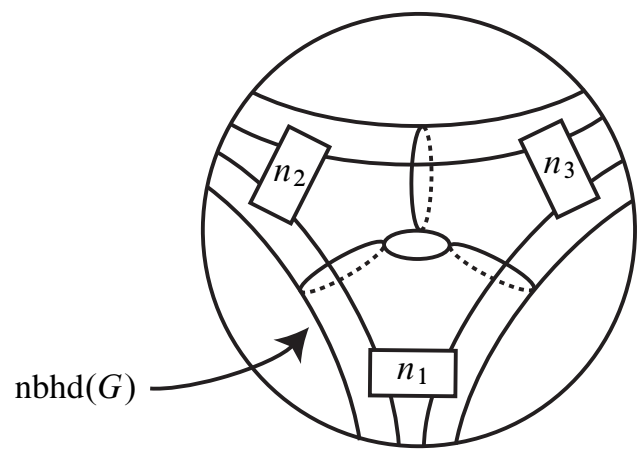

Figure 32

We now show that a solution graph carries a unique solution tangle. By Lemma 3.11 the tangles carried by $G$ are parameterized by integers $n_{1}, n_{2}, n_{3}$ as in Figure 32 (where the boxes are twist boxes). Denote these tangles by $\mathcal{T}\left(n_{1}, n_{2}, n_{3}\right)$. As $G$ is a solution graph, $\mathcal{T}(0,0,0) \cup c_{i}$ will be the 2 -string tangle $1 / f_{i}$, where $f_{i} \in \mathbb{Z}$. Then $\mathcal{T}\left(n_{1}, n_{2}, n_{3}\right) \cup c_{i}$ will be the 2 -string tangle $1 /\left(f_{i}+n_{j}+n_{k}\right)$ where $\{i, j, k\}=\{1,2,3\}$.

Based on Lemma 2.2, we choose $n_{i}, n_{j}, n_{k}$ to satisfy

$$
\begin{aligned}
& n_{1}+n_{2}+f_{3}=-4, \\
& n_{2}+n_{3}+f_{1}=-4, \\
& n_{3}+n_{1}+f_{2}=-4 .
\end{aligned}
$$

Since $f_{1}+f_{2}+f_{3}$ is even, this has a unique integer solution. Then $\mathcal{T}\left(n_{1}, n_{2}, n_{3}\right) \cup c_{i}=$ $1 /-4$ tangle for each $i$. Thus $\mathcal{T}$ is a solution tangle.

The uniqueness of $\mathcal{T}$ follows from the parameterization of the tangles by the $n_{i}$ and the fact that $\mathcal{T} \cup c_{i}=1 /-4$ tangle.

Corollary 3.14 Let $\mathcal{T}$ be an in trans solution tangle, then $\mathcal{T}$ is carried by an in trans solution graph. Conversely, an in trans solution graph carries a unique in trans solution tangle.

Proof Let $\mathcal{T}$ be an in trans solution tangle. Since $\mathcal{T}$ is an in trans solution tangle, $\mathcal{T}$ is a solution tangle. Hence $\mathcal{T}$ is carried by a solution graph, $G$. $G-b_{23}$ corresponds to the graph $s_{12} \cup c_{1} \cup s_{31} \cup e_{1}$. As $\mathcal{T}$ is an in trans solution tangle, $s_{12} \cup c_{1} \cup s_{31}$ lies in a properly embedded disk $D$ in $B^{3}$. Hence $G-b_{23}$ lies in $D$ and is planar. Thus $G$ is an in trans solution graph.

Suppose $G$ is an in trans solution graph. Thus $G$ is a solution graph and carries a solution tangle $\mathcal{T}\left(n_{1}, n_{2}, n_{3}\right)$ (in the notation of the preceding theorem). Since 
$G$ is an in trans solution graph, $G-b_{23}$ is also planar. Then the $2-$ string tangle $\mathcal{T}\left(n_{1}, n_{2}, n_{3}\right)-s_{23}=1 / f$ for some integer $f$. Furthermore, we have chosen $n_{1}, n_{2}, n_{3}$ so that $\ell k\left(x_{i j} \cup s_{i j}, \hat{s}_{i} \cup \hat{x}_{i}\right)=-2$. The argument of Lemma 2.3 shows that $\ell k\left(x_{12} \cup s_{12}, x_{31} \cup s_{31}\right)=-1$. Thus $\mathcal{T}\left(n_{1}, n_{2}, n_{3}\right)-s_{23}=1 /-2$ tangle. Therefore $\mathcal{T}$ is an in trans solution tangle. $\mathcal{T}$ is unique by Theorem 3.13.

Corollary 3.14 allows us to construct infinitely many in trans solution tangles, via in trans solution graphs. Recall that a Brunnian link, $L$, is one such that once any component is removed the remaining components form the unlink. By piping a Brunnian link to a given in trans solution graph, we generate a new in trans solution graph as pictured in Figure 33 (where we have begun with the second solution graph of Figure 30). Similar results hold for solution tangles.

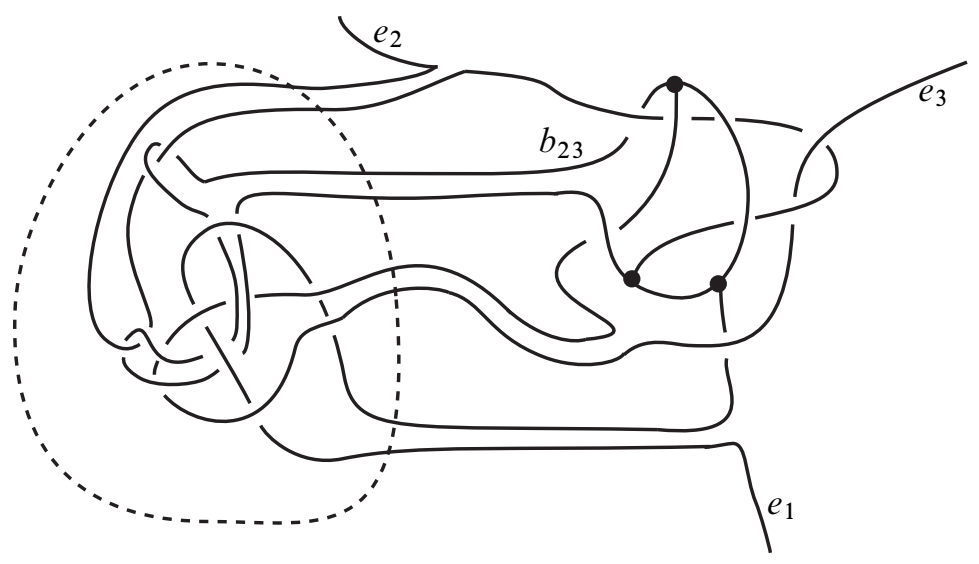

Figure 33

\subsection{Wagon wheel graphs and tetrahedral graphs}

Attaching a 3 -ball, $B^{\prime}$, to the $3-$ ball $B^{3}$ in which $G$ lies, then collapsing $B^{\prime}$ to a point, gives a new graph, $\widehat{G}$, in the 3 -sphere. $\widehat{G}$ is a tetrahedral graph in $S^{3}$, ie a graph abstractly homeomorphic to the 1 -skeleton of a tetrahedron. The edges of $\widehat{G}$ are those of $G: e_{1}, e_{2}, e_{3}, b_{12}, b_{23}, b_{31}$. The planarity of $G$ (ie lying on a disk in $B^{3}$ ) then corresponds to the planarity of $\widehat{G}$ in $S^{3}$ (ie lying on a 2 -sphere in $S^{3}$ ). Also, the exteriors of $G$ and $\widehat{G}$, in $B^{3}$ and $S^{3}$ (resp.), are homeomorphic.

Definition 3.15 A surface $F$ in a 3-manifold $M$ is compressible if there is a disk $D$ embedded in $M$ such that $D \cap F=\partial D$ and $\partial D$ does not bound a disk in $F . F$ is incompressible otherwise (where $F$ is not a 2 -sphere). 
Recall that a graph is abstractly planar if there exists an embedding of it in a 2 -sphere. We are now in a position to use the following characterization of planar graphs in the 3-sphere.

Theorem 3.16 (Thompson [49, Theorem 2.0], see also Scharlemann-Thompson [45]) Let $\widehat{G}$ be an abstractly planar graph embedded in $S^{3} . \widehat{G}$ is planar if and only if

(1) every proper subgraph of $\widehat{G}$ is planar; and

(2) $X(\widehat{G})=S^{3}-\operatorname{nbhd}(\widehat{G})$ has compressible boundary.

Lemma 3.17 Let $e_{n}, n=1,2,3$, be three edges of the tetrahedral graph $\widehat{G}$ which share a common vertex and suppose $\widehat{G}-e_{n}$ is planar for $n=1,2,3$. Suppose there is a fourth edge, $b_{j k}$, such that $X\left(\widehat{G}-b_{j k}\right)$ has compressible boundary. Then $\widehat{G}-b_{j k}$ is a planar graph in $S^{3}$.

Proof We can think of $\widehat{G}-b_{j k}$ as a theta-curve graph, $\widehat{\Theta}$, with two vertices and three edges $e_{i}, e_{j} \cup b_{i j}$, and $e_{k} \cup b_{k i}$. Since $\widehat{G}-e_{n}$ is planar for $n=1,2,3$, the subgraphs $\left(\widehat{G}-b_{j k}\right)-e_{i},\left(\widehat{G}-b_{j k}\right)-\left(e_{j} \cup b_{i j}\right)$, and $\left(\widehat{G}-b_{j k}\right)-\left(e_{k} \cup b_{k i}\right)$ are all planar subgraphs. Since $X\left(\widehat{G}-b_{j k}\right)$ has compressible boundary, $\widehat{G}-b_{j k}$ is a planar graph in $S^{3}$ by Theorem 3.16.

A tetrahedral graph, its corresponding wagon wheel graph, and the tangles carried by the wagon wheel graph are all related by their exterior.

Definition 3.18 For $\mathcal{T}$ a 3 -string tangle with strings $s_{12}, s_{23}, s_{31}$,

$$
X(\mathcal{T})=B^{3}-\operatorname{nbhd}\left(s_{12} \cup s_{23} \cup s_{31} \cup \partial B^{3}\right) .
$$

Lemma 3.19 If $\mathcal{T}$ is carried by the wagon wheel graph $G$ and if $\widehat{G}$ is the corresponding tetrahedral graph, then $X(G)=X(\widehat{G})$ is isotopic to $X(\mathcal{T})$ and $X\left(G-b_{i j}\right)=$ $X\left(\widehat{G}-b_{i j}\right)$ is isotopic to $X\left(\mathcal{T}-s_{i j}\right)$.

Proof See Figure 31.

\subsection{Rational tangles}

$\mathcal{T}$ is rational if there is an isotopy of $B^{3}$ taking $\mathcal{T}$ to a tangle containing no crossings (ie $\mathcal{T}$ is freely isotopic to the standard "jail bar" picture). Rational tangle solutions are generally believed to be the most likely biological models [47; 48] for a number of reasons. As mentioned in the introduction, the short length of captured DNA strands 
implies a simple configuration of entanglement. Furthermore, like 4-plats (2-bridge links), rational tangles look like DNA configurations being built out of plectonemic supercoiling (ie built from sequences of twists). Finally, it is thought in many cases that the DNA winds around the protein surface without crossing itself (eg see Alén et al [1]). Thus, if we push the DNA inside the ball, we have a rational tangle. Below, Corollary 3.21 shows that the only solution tangle among rational tangles is the PJH tangle.

Lemma 3.20 Suppose a rational tangle $\mathcal{T}$ is carried by $G$, then $X(G)$ has compressible boundary and $X\left(G-b_{j k}\right)$ has compressible boundary for all $b_{j k}$.

Proof If $\mathcal{T}$ is rational, then there is a disk, $D$, separating $s_{i j}$ from $s_{i k}$. Thus $X(G)$ has compressible boundary. This disk $D$ also separates the two strands of $\mathcal{T}-s_{j k}$ and thus $X\left(\mathcal{T}-s_{j k}\right)$ has compressible boundary. Since $X\left(\mathcal{T}-s_{j k}\right)$ and $X\left(G-b_{j k}\right)$ are homeomorphic, $X\left(G-b_{j k}\right)$ has compressible boundary.

Corollary 3.21 Suppose $\mathcal{T}$ is rational and $\mathcal{T}$ is a solution tangle, then $\mathcal{T}$ is the PJH tangle.

Proof By Theorem 3.13, $\mathcal{T}$ is carried by a solution graph $G$. Thus, $G-e_{n}$ is planar for $n=1,2,3$. By Lemma 3.20, $X(G)$ has compressible boundary and $X\left(G-b_{j k}\right)$ has compressible boundary for all $b_{j k}$. Lemma 3.19 and Lemma 3.17 imply $\widehat{G}-b_{j k}$ (where $\widehat{G}$ is the tetrahedral graph corresponding to $G$ ) is planar for all $b_{j k}$, and hence $\widehat{G}$ is planar (Theorem 3.16). Thus $G$ is planar. By Lemma 3.12, $\mathcal{T}$ is standard and hence the PJH tangle (Lemma 3.2).

A solution tangle is one that satisfies the three in cis deletion experiments. Thus Corollary 3.21 says that these experiments alone determine the 3-string tangle among rational tangles. It is interesting that this is not true of the inversion experiments. Figure 35 carries a rational tangle that is not the PJH tangle and yet gives the inversion in cis results and the inversion in trans result listed in Table 1 (where the 5 noded knot is the $(2,5)$ torus knot). We would like to thank Jennifer Mann for help in realizing such examples.

Proposition 2.6 allows us to extend the results of this section to experiments in which the in cis deletion products are $(2, p)$ torus links (rather than specifically $(2,4)$ ). For example, the analog of Corollary 3.21 would state that if the synaptic tangle is rational, then three $(2, p)$ torus link in cis products would force it to be standard (plectonemic form) as in Figure 25. 
In contrast to Corollary 3.21, the examples in Figures 34 and 35, as discussed in the proof of Theorem 3.22, show that no other set of three deletion experiments would be enough to determine that a solution $\mathcal{T}$ is standard just from the knowledge that it is rational.

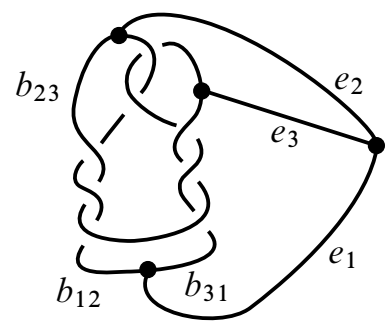

Figure 34

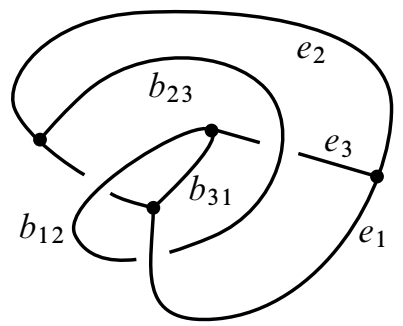

Figure 35

Theorem 3.22 A system of four deletion experiments with $(2, p)$ torus link products must include all three in cis experiments to conclude that a rational tangle satisfying this system is standard.

Proof For the tetrahedral graph in Figure $34, \widehat{G}-e_{1}, \widehat{G}-b_{12}, \widehat{G}-b_{31}, \widehat{G}-b_{23}$ are all planar subgraphs. Deleting a neighborhood of the vertex adjacent to the $e_{i}$ 's results in a wagonwheel graph, $G$. The subgraph $G-e_{2}$ carries a $2-$ string tangle which is not rational. Hence $G-e_{2}$ is not planar and thus $G$ is not planar, yet it does carry a rational tangle. Thus three in trans experiments and one in cis experiments is not sufficient to determine $\mathcal{T}$ is standard under the assumption that $\mathcal{T}$ is rational.

Instead of removing a neighborhood of the vertex adjacent to the $e_{i}$ 's, we can delete a neighborhood of the vertex adjacent to the edges $e_{2}, b_{12}$, and $b_{23}$ from the tetrahedral graph in Figure 34. In this case, we also obtain a wagon wheel graph carrying a rational tangle which is not standard. Hence the nonplanar tetrahedral graph in Figure 34 can also be used to show that two in trans and two in cis experiments, where three of the four corresponding edges in the tetrahedral graph share a vertex, is also not sufficient to determine $\mathcal{T}$ is standard.

The tetrahedral graph in Figure 35 is not planar as $\widehat{G}-e_{2}-b_{31}$ is knotted. However, $\widehat{G}-e_{1}, \widehat{G}-e_{3}, \widehat{G}-b_{23}$, and $\widehat{G}-b_{12}$ are all planar. Hence this graph shows that the remaining case, two in trans and two in cis experiments where no three of the four corresponding edges in the tetrahedral graph share a common vertex, is also not sufficient to determine $\mathcal{T}$ is standard. 


\subsection{The exterior $X(G)$}

Our goal is to prove Corollary 3.32, which extends Corollary 3.21. In Section 4, we will use Corollary 3.32 to show there is a unique small crossing solution tangle. We first establish some properties of the exterior of both solution graphs and in trans solution graphs.

Definition 3.23 Let $M$ be a 3-manifold, $F$ a subsurface of $\partial M$, and $J$ a simple closed curve in $F$. Then $\tau(M, J)$ is the 3 -manifold obtained by attaching a 2handle to $M$ along $J$. That is, $\tau(M, J)=M \cup_{J} H$, where $H$ is a 2-handle. $\sigma(F, J)$ is the subsurface of $\partial \tau(M, J)$ obtained by surgering $F$ along $\partial H$. That is, $\sigma(F, J)=(F \cup \partial H)-\operatorname{int}(F \cap \partial H)$.

Handle Addition Lemma $[29 ; 11 ; 44 ; 56]$ Let $M$ be an orientable, irreducible 3 -manifold and $F$ a surface in $\partial M$. Let $J$ be a simple, closed curve in $F$. Assume $\sigma(F, J)$ is not a 2 -sphere. If $F$ is compressible in $M$ but $F-J$ is incompressible, then $\sigma(F, J)$ is incompressible in $\tau(M, J)$.

Lemma 3.24 Let $G$ be a wagon wheel graph and $X(G)$ its exterior. Let $J_{i}$ and $\gamma_{i j}$ be the meridian curves on $\partial X(G)$ as in Figure 29 (and defined right above the figure). Suppose $\partial X(G)$ compresses in $X(G)$. If $G$ is a solution graph, then $\partial X(G)-$ $\left(J_{1} \cup J_{2} \cup J_{3}\right)$ is compressible in $X(G)$. If $G$ is an in trans solution graph, then $\partial X(G)-\left(\gamma_{23} \cup J_{1} \cup J_{2} \cup J_{3}\right)$ is compressible in $X(G)$.

Proof Assume $\partial X(G)$ compresses in $X(G)$. Note that $X(G)$ is irreducible. We will use the following four claims to prove this lemma:

Claim 3.25 If $G-b_{23}$ is planar (eg $G$ is an in trans solution graph), then $\partial X(G)-\gamma_{23}$ is compressible in $X(G)$.

Proof Since $G-b_{23}$ is planar, $\tau\left(X(G), \gamma_{23}\right)=X\left(G-b_{23}\right)$ has compressible boundary. The claim now follows from the Handle Addition Lemma.

If $G$ is an in trans solution graph, let $F_{1}=\partial X(G)-\gamma_{23}$. By Claim 3.25, $F_{1}$ is compressible in $X(G)$. Else if $G$ is a solution graph, let $F_{1}=\partial X(G)$. In this case, $F_{1}$ is compressible in $X(G)$ by hypothesis.

Claim 3.26 $F_{2}=F_{1}-J_{1}$ is compressible in $X(G)$. 


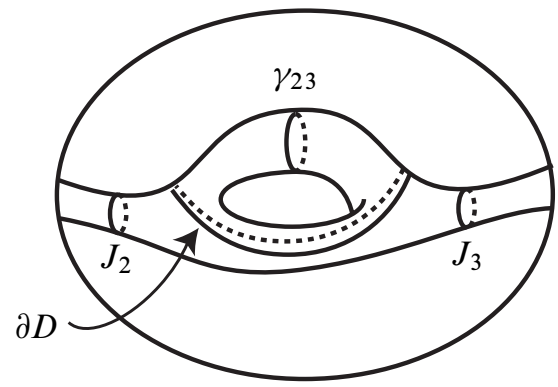

$D=$ compressing disk

Figure 36

Proof $F_{1}$ is compressible in $X(G)$. On the other hand, since $\tau\left(X(G), J_{1}\right)=$ $X\left(G-e_{1}\right)$ and $G-e_{1}$ is planar, $\sigma\left(F_{1}, J_{1}\right)$ compresses in $\tau\left(X(G), J_{1}\right)$. See Figure 36. The claim now follows from the Handle Addition Lemma.

Claim 3.27 $\quad F_{3}=F_{2}-J_{2}$ is compressible in $X(G)$.

Proof Since $G-e_{2}$ is planar, $\sigma\left(F_{2}, J_{2}\right)$ is compressible in $\tau\left(X(G), J_{2}\right)$ (as in the analog of Figure 36). Thus Claim 3.26 with the Handle Addition Lemma implies the claim.

Claim 3.28 $F=F_{3}-J_{3}$ is compressible in $X(G)$.

Proof $G-e_{3}$ is planar, hence $\sigma\left(F_{3}, J_{3}\right)$ is compressible in $\tau\left(X(G), J_{3}\right)=X\left(G-e_{3}\right)$ (as in the analog of Figure 36). Claim 3.27 with the Handle Addition Lemma says that $F_{3}-J_{3}$ is compressible in $X(G)$.

Thus if $G$ is a solution graph, then $F=\partial X(G)-\left(J_{1} \cup J_{2} \cup J_{3}\right)$ is compressible in $X(G)$. If $G$ is an in trans solution graph, then $F=\partial X(G)-\left(\gamma_{23} \cup J_{1} \cup J_{2} \cup J_{3}\right)$ is compressible in $X(G)$. This completes the proof of Lemma 3.24.

\subsection{Solution graphs}

Lemma 3.29 Let $G$ be a solution graph and $X(G)$ its exterior. Let $J_{i}$ and $\gamma_{i j}$ be the meridian curves on $\partial X(G)$ as in Figure 29 (and defined right above the figure). If $\partial X(G)$ compresses in $X(G)$, then there is a properly embedded disk in $X(G)$ that intersects each $\gamma_{i j}$ algebraically once in $\partial X(G)$ and is disjoint from the $J_{i}$. 
Proof Let $D^{\prime}$ be a compressing disk, guaranteed by Lemma 3.24, properly embedded in $X(G)$ such that $\partial D^{\prime} \subset F=\partial X(G)-\left(J_{1} \cup J_{2} \cup J_{3}\right)$ and $\partial D^{\prime}$ does not bound a disk in $F$. Write $B^{3}=X(G) \cup_{\partial X(G)} M$ where $M=\operatorname{nbhd}\left(G \cup \partial B^{3}\right)$. In the notation of Figure 29, let $M-\operatorname{nbhd}\left(\mathcal{J}_{1} \cup \mathcal{J}_{2} \cup \mathcal{J}_{3}\right)=M_{1} \cup M_{2}$ where $M_{1}$ is $S^{2} \times I$ and $M_{2}$ is a solid torus. If $\partial D^{\prime}$ lies in $\partial M_{1}$, we can form a 2 -sphere, $S$, by capping off $D^{\prime}$ with a disk in $M_{1}$ (take the disk bounded by $\partial D^{\prime}$ on $\partial M_{1}$ and push in slightly). That is, $S$ is a 2-sphere which intersects $\partial X(G)$ in $\partial D^{\prime}$. But then $S$ would have to be nonseparating (there would be an arc from $\mathcal{J}_{1}$ to $\mathcal{J}_{2}$, say, on $\partial M_{1}$ which intersected $\partial D^{\prime}$ once and an arc connecting $\mathcal{J}_{1}$ to $\mathcal{J}_{2}$ on $\partial M_{2}$ which missed $\partial D^{\prime}$, hence $S$, altogether). But 2 -spheres in a 3-ball are separating.

Thus $\partial D^{\prime}$ must lie in $\partial M_{2}$. If $\partial D^{\prime}$ is essential in $\partial M_{2}$, then $\partial D^{\prime}$ must intersect any meridian of the solid torus $M_{2}$ algebraically once (else $B^{3}$ would contain a lens space summand). Then $D^{\prime}$ is the desired disk, and we are done. So assume $\partial D^{\prime}$ bounds a disk, $D^{\prime \prime}$, in $\partial M_{2}$. Then the disks $\operatorname{nbhd}\left(\mathcal{J}_{1} \cup \mathcal{J}_{2} \cup \mathcal{J}_{3}\right) \cap \partial M_{2}$ must lie in $D^{\prime \prime}$, as $S=D^{\prime} \cup D^{\prime \prime}$ is separating. Since $G-e_{1}$ is planar, $M_{2}$ is an unknotted solid torus in $B^{3}$. Thus there is a properly embedded disk $D \subset B^{3}-\operatorname{int} M_{2}$ such that $\partial D$ is a longitude of $\partial M_{2}$ and hence is essential. Furthermore, we can arrange that $D$ is disjoint from $D^{\prime \prime}$. Then $D \cap S$ is a collection of trivial circles in $D^{\prime}$ and $D$ can be surgered off $S$ without changing $\partial D$. In particular, $D$ is properly embedded in $X(G)$ since the $e_{i}$ lie on the opposite side of $S$ from $D . D$ is the desired disk.

Definition 3.30 Let $\mathcal{T}$ be a 3-string tangle. $\mathcal{T}$ is split if there is a properly embedded disk separating two of its strands from the third (Figure 37 (a)). Strands $s_{1}, s_{2}$ of $\mathcal{T}$ are parallel if there is a disk $D$ in $B^{3}$ such that $\operatorname{int}(D)$ is disjoint from $\mathcal{T}$ and $\partial D=s_{1} \cup \alpha \cup s_{2} \cup \beta$ where $\alpha, \beta$ are arcs in $\partial B^{3}$ (Figure 37 (b)). Note: Rational

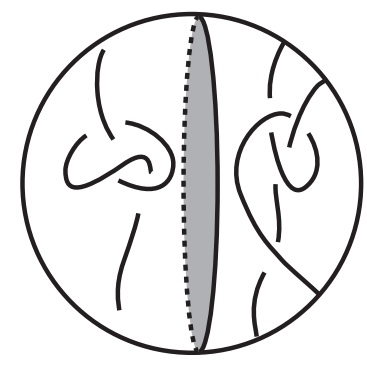

(a) split

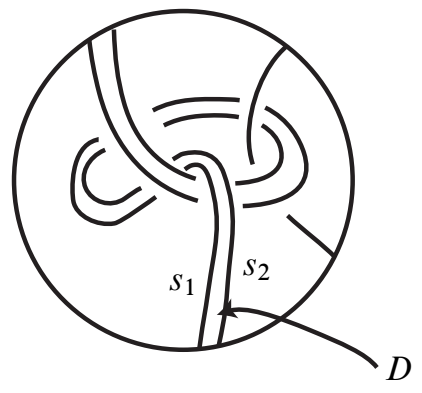

(b) parallel

Figure 37

tangles are split and have parallel strands. 
Lemma 3.31 Let $\mathcal{T}$ be a 3-string tangle carried by a solution graph $G$. If $\mathcal{T}$ is rational, split, or if $\mathcal{T}$ has parallel strands, then $X(G)$ and $X\left(G-b_{i j}\right)$, for each $i, j$, have compressible boundary.

Proof If $\mathcal{T}$ is rational, split, or has parallel strands, then $\partial X(\mathcal{T})$ is compressible in $X(\mathcal{T})$. By Lemma 3.19, $\partial X(G)$ is compressible in $X(G)$.

We now argue that $\partial X\left(G-b_{i j}\right)$ is compressible in $X\left(G-b_{i j}\right)$. Below $\{i, j, k\}=$ $\{1,2,3\}$.

If $\mathcal{T}$ is split, then there is a disk, $D$, separating one strand from two. If $s_{i j}$ is on the side of $D$ containing two, then $D$ still separates the two strands of $\mathcal{T}-s_{i j}$ and $X\left(\mathcal{T}-s_{i j}\right)$ still has compressible boundary. By Lemma 3.19, $X\left(G-b_{i j}\right)$ has compressible boundary. So we assume $s_{i j}$ is on one side of $D$ and $s_{j k}, s_{k i}$ on the other. As a disk properly embedded in $X(G), D$ separates the curves $\gamma_{i j}, \gamma_{j k}$ of $\partial X(G)$. By Lemma 3.29 there is a disk $D^{\prime}$ in $X(G)$ that intersects $\gamma_{j k}$ on $\partial X(G)$ algebraically a nonzero number of times. After surgering along $D$ (while preserving the nonzero algebraic intersection with $\gamma_{j k}$ ), we may assume that $D^{\prime}$ is disjoint from $D$, and hence from $\gamma_{i j}$. But then $D^{\prime}$ is a compressing disk for $G-b_{i j}$.

If $\mathcal{T}$ has parallel strands, we argue in two cases according to which strands are parallel: First, assume $s_{j k}, s_{k i}$ are parallel. Then they are parallel in $\mathcal{T}-s_{i j}$ and $X\left(\mathcal{T}-s_{i j}\right)$ has compressible boundary. By Lemma 3.19, $X\left(G-b_{i j}\right)$ has compressible boundary.

So, assume that $s_{i j}$ and $s_{j k}$ are parallel. Then there is a disk, $D$, properly embedded in $X(G)$ that intersects $\gamma_{i j}$ exactly once, $\gamma_{j k}$ exactly once, and is disjoint from $\gamma_{k i}$. By Lemma 3.29, there is also a disk, $D^{\prime}$, in $X(G)$ that intersects $\gamma_{k i}$ algebraically once. After possibly surgering $D^{\prime}$ along $D$ we may assume the $D$ and $D^{\prime}$ are disjoint. But $X(G)-\operatorname{nbhd}(D)$ is homeomorphic to $X\left(G-b_{i j}\right)$, and $D^{\prime}$ becomes a compressing disk in $X\left(G-b_{i j}\right)$.

Corollary 3.32 Let $\mathcal{T}$ be a solution tangle. If $\mathcal{T}$ is rational or split or if $\mathcal{T}$ has parallel strands, then $\mathcal{T}$ is the PJH tangle.

Proof Let $G$ be a solution graph carrying $\mathcal{T}$. Then Lemmas 3.31, 3.19, and 3.17 imply that $\widehat{G}-b_{j k}$ planar for all $b_{j k}$ where $\widehat{G}$ is the associated tetrahedral graph. Thus Theorem 3.16 implies $\widehat{G}$ is planar. Thus $G$ is planar. By Lemma $3.12, \mathcal{T}$ is standard. Now Lemma 3.2 says $\mathcal{T}$ is the PJH tangle.

Remark Since in trans solution tangles are also solution tangles, Corollary 3.32 also applies to in trans solution tangles. Although the three in cis deletion equations suffice 
to determine $\mathcal{T}$ if $\mathcal{T}$ is rational, split or has parallel strands, the in trans deletion experiment does rule out some exotic solutions. For example, the tangle in Figure 38 satisfies all the in cis experimental results (ie it is a solution tangle), but does not satisfy the in trans results (ie it is not an in trans solution tangle).
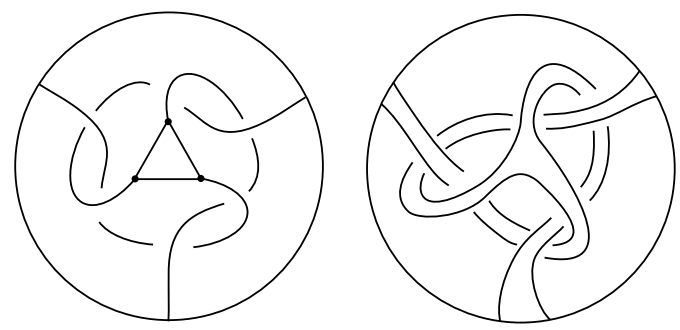

Figure 38

\subsection{In trans solution graphs}

In Lemma 3.33 we will show that the exterior of an in trans solution graph is a handlebody. This need not be true for solution graphs. The solution graph of Figure 38 contains an incompressible genus 2 surface in its exterior (it is based on Thurston's tripos graph). Thus its exterior is not a handlebody. We will also use Lemma 3.33 to further explore planarity of tetrahedral graphs in the next section.

Lemma 3.33 Let $G$ be an in trans solution graph and $X(G)$ its exterior. Let $J_{i}$ and $\gamma_{i j}$ be the meridian curves on $\partial X(G)$ as in Figure 29 (and defined right above the figure). If $\partial X(G)$ compresses in $X(G)$, then there is a properly embedded disk in $X(G)$ that intersects $\gamma_{23}$ exactly once, intersects each of $\gamma_{12}, \gamma_{31}$ algebraically once, and is disjoint from the $J_{i}$. Furthermore, $X(G)$ is a handlebody.

Proof By Lemma 3.24, there is a disk $D^{\prime}$ properly embedded in $X(G)$ such that $\partial D^{\prime} \subset F=\partial X(G)-\left(\gamma_{23} \cup J_{1} \cup J_{2} \cup J_{3}\right)$ and $\partial D^{\prime}$ does not bound a disk in $F$. Write $B^{3}=X(G) \cup_{\partial X(G)} M$ where $M=\operatorname{nbhd}\left(G \cup \partial B^{3}\right)$. In the notation of Figure 29, let $M-\operatorname{nbhd}\left(\mathcal{J}_{1} \cup \mathcal{J}_{2} \cup \mathcal{J}_{3} \cup \Gamma_{23}\right)=M_{1} \cup M_{2}$ where $M_{1}$ is $S^{2} \times I$ and $M_{2}$ is a 3-ball. $\partial D^{\prime}$ lies in $\partial M_{i}$ for some $i$, hence we can form a 2-sphere, $S$, by capping off $D^{\prime}$ with a disk in $M_{i}$ (take the disk bounded by $\partial D^{\prime}$ on $\partial M_{i}$ and push in slightly). That is, $S$ is a 2-sphere which intersects $\partial X(G)$ in $\partial D^{\prime}$. If $\partial D^{\prime}$ lay on $\partial M_{1}$, then $S$ would have to be nonseparating. But $2-$ spheres in a 3 -ball are separating. Thus $\partial D^{\prime}$ must lie in $\partial M_{2}$. But then in $\partial M_{2}$, the disks $\operatorname{nbhd}\left(\mathcal{J}_{1} \cup \mathcal{J}_{2} \cup \mathcal{J}_{3}\right) \cap \partial M_{2}$ must lie on opposite sides of $\partial D^{\prime}$ from the disks $\Gamma_{23}^{\prime} \cup \Gamma_{23}^{\prime \prime}=\operatorname{nbhd}\left(\Gamma_{23}\right) \cap \partial M_{2}$, as $S$ 
is separating. Thus $S$ can be isotoped to intersect $\operatorname{nbhd}(G)$ in the curve gotten by connecting the two disks $\Gamma_{23}^{\prime}, \Gamma_{23}^{\prime \prime}$ by a band $d$ lying in $\partial \operatorname{nbhd}(G)$. See Figure 39 . We may view $S$ as a $2-$ sphere which intersects $G$ in only two points and separates

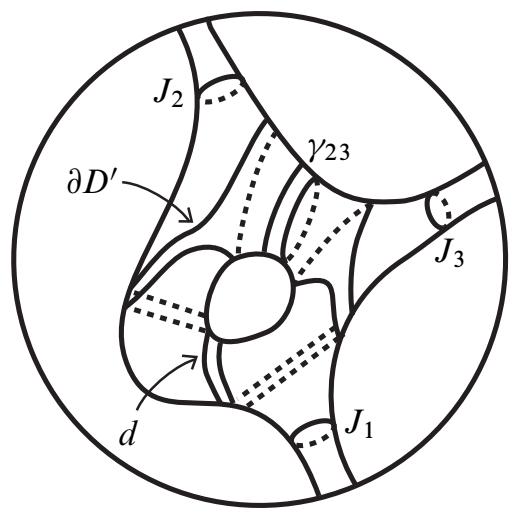

Figure 39

off a subarc $b_{23}^{\prime}$ of $b_{23}$. Now $b_{23}^{\prime}$ must be unknotted in the 3 -ball bounded by $S$, otherwise deleting $e_{1}$ would not give a planar graph. Hence, there exists a disk $D$ in the 3-ball bounded by $S$ such that the boundary of $D$ consists of the arc $b_{23}^{\prime}$ and an arc in $\Gamma_{23}^{\prime} \cup \Gamma_{23}^{\prime \prime} \cup d$. Then $\mathrm{D}$ can be taken to be a disk, properly embedded in $X(G)$ whose boundary is disjoint from $J_{1} \cup J_{2} \cup J_{3}$ and whose boundary intersects $\gamma_{23}$ exactly once. Finally, as $\gamma_{12}, \gamma_{31}$ are isotopic to $\gamma_{23}$ on the boundary of the solid torus component of $M-\operatorname{nbhd}\left(\mathcal{J}_{1} \cup \mathcal{J}_{2} \cup \mathcal{J}_{3}\right), \partial D$ intersects these curves algebraically once. Furthermore, $X(G)-\operatorname{nbhd}(D)$ is homeomorphic to $X\left(G-\gamma_{23}\right)$, which is a handlebody. Thus $X(G)$ is a handlebody.

\subsection{Tetrahedral graph planarity}

In the above model, each mathematical assumption about the planarity of the in trans solution graph after deleting an edge corresponds to an experimental result. For the economy of experiment, then, it is natural to ask how much information is necessary to conclude the planarity of the graph. Recall that by collapsing the outside 2-sphere to a vertex, a wagon wheel graph in $B^{3}$ becomes a tetrahedral graph $\widehat{G}$ in $S^{3}$. We discuss this issue of economy in the context of tetrahedral graphs and in the spirit of Theorem 3.16 (see also Scharlemann [45], Gordon [22], Wu [57] and Robertson [40]). In particular, Theorem 3.34 and Corollary 3.37 say that, in contrast to Theorem 3.16, to check the planarity of a tetrahedral graph, one does not need to check the planarity of all subgraphs. We end with examples showing that Corollary 3.37 is sharp. 
Theorem 3.34 Let $\widehat{G}$ be a tetrahedral graph embedded in $S^{3}$. Then $\widehat{G}$ is planar if and only if

(1) the exterior of $\widehat{G}$ has compressible boundary; and

(2) there is an edge, $\epsilon^{\prime}$, of $\widehat{G}$ such that for any edge $e \neq \epsilon^{\prime}$ of $\widehat{G}, \widehat{G}-e$ is planar.

Proof If $\widehat{G}$ is planar then clearly the two conditions hold. We prove the converse: the two listed conditions on $\widehat{G}$ guarantee that $\widehat{G}$ is planar. Let $\epsilon$ be the edge of $\widehat{G}$ which does not share an endpoint with $\epsilon^{\prime}$. Let $e_{1}, e_{2}, e_{3}, e_{4}$ be the four edges of $\widehat{G}$ other than $\epsilon, \epsilon^{\prime}$. Let $d_{i}$ be the meridian disk corresponding to the edge $e_{i}$ in $\operatorname{nbhd}(\widehat{G})$ and let $m_{i}=\partial d_{i}$ be the corresponding meridian curve on the boundary of $X(\widehat{G})$, the exterior of $\hat{G}$.

Claim 3.35 $X(\widehat{G})$ is a handlebody.

Proof Pick a vertex, $v$, of $\widehat{G}$ not incident to $\epsilon^{\prime}$. Then removing a neighborhood of $v$ from $S^{3}, \widehat{G}$ becomes an in trans solution graph $G$ in a 3 -ball. Furthermore, $X(G)$ is homeomorphic to $X(\widehat{G})$. The Claim now follows from Lemma 3.33.

Recall the following from [22].

Theorem 3.36 Let $\mathcal{C}$ be a set of $n+1$ disjoint simple loops in the boundary of a handlebody $X$ of genus $n$, such that $\tau\left(X ; \mathcal{C}^{\prime}\right)$ is a handlebody for all proper subsets $\mathcal{C}^{\prime}$ of $\mathcal{C}$. Then $\cup \mathcal{C}$ bounds a planar surface $P$ in $\partial X$ such that $(X, P) \cong(P \times I, P \times 0)$, where $I=[0,1]$.

Set $X=X(\widehat{G})$ and $\mathcal{C}=\left\{m_{1}, m_{2}, m_{3}, m_{4}\right\}$. Since $\widehat{G}-e_{i}$ is planar for $i=1, \ldots, 4$, Theorem 3.36 implies that there is a planar surface $P$ in $X(\widehat{G})$ whose boundary is $\left\{m_{1}, m_{2}, m_{3}, m_{4}\right\}$ and such that $(X(\widehat{G}), P) \cong(P \times I, P \times 0)$. Capping $P \times\{1 / 2\}$ with the meridian disks $\left\{d_{1}, d_{2}, d_{3}, d_{4}\right\}$ gives a 2 -sphere $\widehat{P}$ in $S^{3}$ disjoint from $\widehat{G}$ except for a single point intersection with each of the edges $e_{1}, e_{2}, e_{3}, e_{4}$. Let $\widehat{G}-\widehat{P}=G_{1} \cup G_{2}$. Labeling the edges as in $\widehat{G}$, we take $G_{1}$ with edges $\left\{\epsilon, e_{1}, e_{2}, e_{3}, e_{4}\right\}$ and $G_{2}$ with edges $\left\{\epsilon^{\prime}, e_{1}, e_{2}, e_{3}, e_{4}\right\}$. Furthermore, we may label so that $e_{1}, e_{2}$ share a vertex in $G_{1}$ and $e_{2}, e_{4}$ share a vertex in $G_{2}$. See Figure 40.

Let $N_{1}=\operatorname{nbhd}\left(G_{1}\right)$ and $N_{2}=\operatorname{nbhd}\left(G_{2}\right)$. Then $\operatorname{nbhd}(\widehat{G})$ is the union of $N_{1}$ and $N_{2}$ along the meridian disks $\left\{d_{1}, d_{2}, d_{3}, d_{4}\right\}$. Now the pair $\left(N_{i}, \cup d_{j}\right)=\left(F_{i} \times I, \cup \alpha_{i}^{j} \times I\right)$ where $i \in\{1,2\}, j \in\{1,2,3,4\}$ and where each $F_{i}$ is a disk, each $\alpha_{i}^{j}$ is an arc in $\partial F_{i}$. Each $G_{i}$ can be taken to lie in $F_{i} \times\{1 / 2\}$. Write $S^{3}=B_{1} \cup \widehat{P} B_{2}$ where the $B_{i}$ are 3 -balls. Because $(X(\widehat{G}), P) \cong(P \times I, P \times 0)$, we may extend $F_{i} \times\{1 / 2\}$ to a 


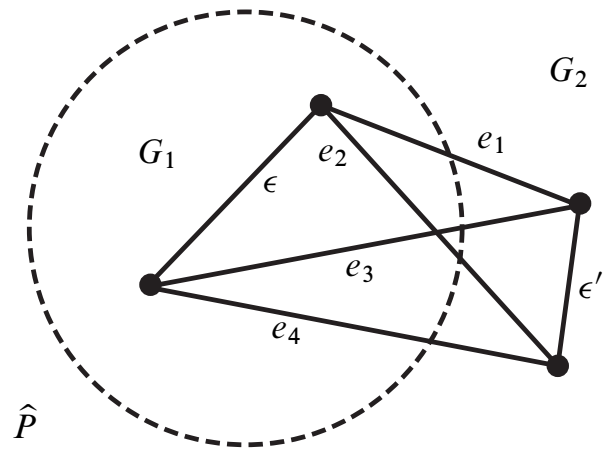

Figure 40
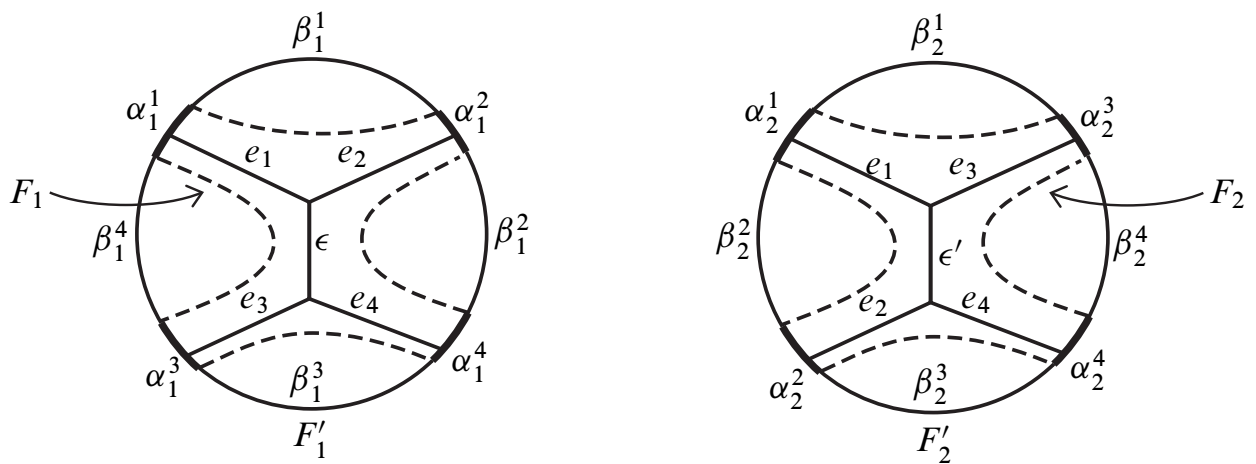

Figure 41

properly embedded disk $F_{i}^{\prime}$ in $B_{i}$ containing $G_{i}$. By a proper choice of the initial $F_{i}$ we may assume that $F_{i}^{\prime}-\left(\cup \alpha_{i}^{j}\right)$ is a union of four arcs $\beta_{i}^{j}$, labelled as in Figure 41 . In particular,

- $\beta_{1}^{1}$ is isotopic to $e_{1} \cup e_{2}$ and $\beta_{1}^{3}$ is isotopic to $e_{3} \cup e_{4}$ in $B_{1}$ (keeping the endpoints in $\cup d_{j}$ on $\partial B_{1}=\widehat{P}$ ), and

- $\beta_{2}^{1}$ is isotopic to $e_{1} \cup e_{3}$ and $\beta_{2}^{3}$ is isotopic to $e_{2} \cup e_{4}$ in $B_{2}$ (keeping the endpoints in $\cup d_{j}$ on $\partial B_{1}=\widehat{P}$ ).

The boundary of $F_{1}^{\prime}$ frames the 4-punctured sphere $P \subset \widehat{P}$. This allows us to assign a rational number slope on properly embedded arcs in $P$ (up to proper isotopy in $P$ ). We take $\beta_{1}^{1}, \beta_{1}^{3}$ to have slope $0 / 1$ and $\beta_{1}^{2}, \beta_{1}^{4}$ to have slope $1 / 0$. It follows that $\beta_{2}^{1}, \beta_{2}^{3}$ must have slope $1 / n$ : otherwise the edges $e_{1}, e_{2}, e_{3}, e_{4}$ of $\widehat{G}$ will form a nontrivial $2-$ bridge knot, but we are assuming that $\widehat{G}-\epsilon$ is planar. 
By rechoosing $F_{1}$ we may assume that $\beta_{1}^{2}$ is $\beta_{2}^{3}$ and that $\beta_{1}^{4}$ is $\beta_{2}^{1}$. Then rechoosing $F_{2}$ (by twisting along the disk disjoint from $\beta_{2}^{1} \cup \beta_{2}^{3}$ in $B_{2}$ ), we may further take $\beta_{2}^{2}$ to be $\beta_{1}^{1}$ and $\beta_{2}^{4}$ to be $\beta_{1}^{3}$. Then $F_{1}^{\prime} \cup F_{2}^{\prime}$ is a 2 -sphere in $S^{3}$ containing $\hat{G}$ (Alternatively, instead of rechoosing $F_{2}$, after rechoosing $F_{1}$ so that $\beta_{1}^{2}=\beta_{2}^{3}$ and $\beta_{1}^{4}=\beta_{2}^{1}$, we can extend $F_{1}$ to a disk containing $\widehat{G}-\epsilon^{\prime}$. Thus $\widehat{G}-\epsilon^{\prime}$ is planar and $\widehat{G}$ is planar by Theorem 3.16). Thus we have proved Theorem 3.34.

We will now use Theorem 3.34 to prove Corollary 3.37.

Corollary 3.37 Suppose $\widehat{G}$ is a tetrahedral graph embedded in $S^{3}$ with the following properties:

(1) There exists three edges $\epsilon_{1}, \epsilon_{2}, \epsilon_{3}$ such that $\widehat{G}-\epsilon_{i}$ is planar.

(2) The three edges $\epsilon_{1}, \epsilon_{2}, \epsilon_{3}$ share a common vertex.

(3) There exists two additional edges, $\epsilon_{4}$ and $\epsilon_{5}$ such that $X\left(\widehat{G}-\epsilon_{4}\right)$ and $X\left(\widehat{G}-\epsilon_{5}\right)$ have compressible boundary.

(4) $X(\widehat{G})$ has compressible boundary.

Then $\widehat{G}$ is planar.

Proof By Lemma 3.17, $\widehat{G}-\epsilon_{4}$ and $\widehat{G}-\epsilon_{5}$ are planar. Thus by Theorem 3.34, $\widehat{G}$ is planar.

We finish this section with examples that show that if any single hypothesis of Corollary 3.37 is dropped then its conclusion, that $\widehat{G}$ is planar, may not hold. The tetrahedral graph in Figure 35 is not planar as $\widehat{G}-e_{2}-b_{31}$ is knotted. However, $\widehat{G}-e_{1}, \widehat{G}-e_{3}$, $\widehat{G}-b_{23}$, and $\widehat{G}-b_{12}$ are all planar. Furthermore, $X(\widehat{G})$ is a handlebody as the edge $b_{23}$ can be isotoped onto a neighborhood of $\widehat{G}-b_{23}$. (Alternatively, we can determine that $X(\widehat{G})$ is a handlebody by removing the vertex adjacent to the $e_{n}$ 's, creating a wagon wheel graph, $G$, which carries a rational tangle $\mathcal{T}$. Since $X(\widehat{G})=X(G)=X(\mathcal{T})$ and $\mathcal{T}$ is rational, $X(\widehat{G})$ is a handlebody.) Thus $X(\widehat{G})$ has compressible boundary. $X\left(\widehat{G}-b_{31}\right)$, and $X\left(\widehat{G}-e_{2}\right)$ also have compressible boundary. Thus properties (1), (3), (4) in Corollary 3.37 hold, but property 2 does not hold.

In Figure 34, $\widehat{G}-e_{1}, \widehat{G}-b_{12}, \widehat{G}-b_{31}, \widehat{G}-b_{23}$ are all planar graphs. The edges $e_{1}, b_{12}$, and $b_{31}$ share a common vertex. Since $\widehat{G}-b_{23}$ is planar, $X\left(\widehat{G}-b_{23}\right)$ has compressible boundary. Removing the vertex adjacent to the $e_{n}$ 's in $\widehat{G}$ results in a wagon wheel graph, $G$, which carries a rational tangle. By Lemma $3.19 X(G)$ is a handlebody, and thus $X(\widehat{G})$ has compressible boundary. On the other hand, $G-e_{2}, G-e_{3}$ 
carry tangles which are nontrivial disk sums that do not have compressible boundary (by Lemma 3.3 of [58]). By Lemma 3.19, neither $X\left(G-e_{2}\right)$ nor $X\left(G-e_{3}\right)$ have compressible boundary. Hence neither $X\left(\widehat{G}-e_{2}\right)$ nor $X\left(\widehat{G}-e_{3}\right)$ have compressible boundary. In particular, $\widehat{G}$ is not planar. Thus the tetrahedral graph in Figure 34 satisfies properties (1), (2), (4) in Corollary 3.37, but only satisfies half of property $3\left(X\left(\widehat{G}-b_{23}\right)\right.$ has compressible boundary, but there does not exist a fifth such edge).

Note that the tangle, $\mathcal{T}$, in Figure 42 is carried by a nonplanar wagon wheel graph, $G$. If $G$ were planar, then $\mathcal{T}$ would be the PJH tangle by Lemmas 3.12 and 3.2. To see

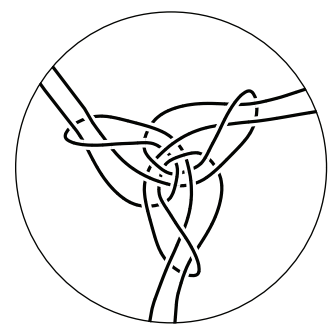

Figure 42

that $\mathcal{T}$ is not the PJH tangle, cap off these tangles by arcs along the tangle circle that are complementary to the $c_{i}$ (ie the $x_{i j}$ of Figure 13) to form 3 component links. These two 3-component links are not isotopic. For example, they have different hyperbolic volumes as computed by SnapPea. Let $\widehat{G}$ be the tetrahedral graph corresponding to $G$. Every subgraph of $\widehat{G}$ is planar. Hence conditions (1), (2), (3) of Corollary 3.37 hold, but $X(\widehat{G})$ does not have compressible boundary (by Theorem 3.16).

\section{If $\mathcal{T}$ is not the $\mathrm{PJH}$ tangle, then $\operatorname{Cr}(\mathcal{T}) \geq 8$ up to free isotopy}

Definition 4.1 Two 3-tangles $\mathcal{T}_{1}, \mathcal{T}_{2}$ are freely isotopic if there is an isotopy of the 3-ball, which is not necessarily fixed on its boundary, taking $\mathcal{T}_{1}$ to $\mathcal{T}_{2}$.

In the last section we showed that if a solution tangle is split or has two parallel strands, then it must be the PJH tangle. Here we show that if a tangle can be freely isotoped to have fewer than eight crossings, then it is either split or has two parallel strands. Hence a solution tangle which can be freely isotoped to have fewer than eight crossings must be the PJH tangle (Corollary 4.8). As a rational tangle is one which can be freely isotoped to have no crossings, one can think of Corollary 4.8 as a generalization of the result (Corollary 3.32) that the only rational solution tangle is the PJH tangle. 
Corollary 4.8 also gives a lower bound on the crossing number of an exotic solution tangle. Using the in trans deletion result, we will improve this lower bound in the next section after imposing a normal framing on the tangle (working with tangle equivalence rather than free isotopy).

Lemma 4.2 If one string of a 3-tangle $\mathcal{T}$ crosses the union of the other two strings at most once, then $\mathcal{T}$ is split.

Proof If the string passes over the union, isotop the strand to the front hemisphere of the tangle sphere, otherwise isotop to the back.

Definition 4.3 To a projection of a 3 -string tangle $\mathcal{T}$ we associate the 4 -valent graph $\Gamma(\mathcal{T})$, that is obtained by placing a vertex at each crossing. If $\mathcal{T}$ is not split, we label in sequence $e_{1}, \ldots, e_{6}$ the distinct edges which are incident to the tangle circle. Let $v_{1}, \ldots, v_{6}$ be the vertices of $\Gamma(\mathcal{T})$ which are endpoints of $e_{1}, \ldots, e_{6}$ as in Figure 43.



Figure 43

Lemma 4.4 Assume $\mathcal{T}$ is not split. If $v_{i}=v_{j}$ for some $i \neq j$, then the crossing number of its projection can be reduced by free isotopy.

Proof Assume $v_{i}=v_{j}$ with $i \neq j$. If $e_{i}, e_{j}$ are opposing at $v_{i}$, then $e_{i} \cup e_{j}$ is a string of $\mathcal{T}$ intersecting the other strings exactly once. By Lemma 4.2, $\mathcal{T}$ is split. So we assume $e_{i}, e_{j}$ are not opposing at $v_{i}$, as in Figure 44.



Figure 44

But then we can untwist $e_{i}, e_{j}$ to reduce the crossing number. 
Definition 4.5 If $\mathcal{T}$ is not split, let $f_{i}$ be the face of $\Gamma(\mathcal{T})$ containing $e_{i}, e_{i+1}$.

Lemma 4.6 Assume $\mathcal{T}$ is not split. No two edges of $f_{i}$ correspond to the same edge of $\Gamma(\mathcal{T})$. If two vertices of $f_{i}$ correspond to the same vertex of $\Gamma(\mathcal{T})$, then the crossing number of the projection can be reduced by an isotopy fixed on the boundary. Finally, if $v_{j}$ is incident to $f_{i}$, then $j \in\{i, i+1\}$ or a crossing can be reduced by a free isotopy.

Proof Assume that two edges of $f_{i}$ correspond to the same edge of $\Gamma(\mathcal{T})$ (Figure 45). Then there would be a circle in the interior of the tangle circle intersecting the edge once. Then a string of $\mathcal{T}$ intersects this circle exactly once contradicting the Jordan Curve Theorem.



Figure 45

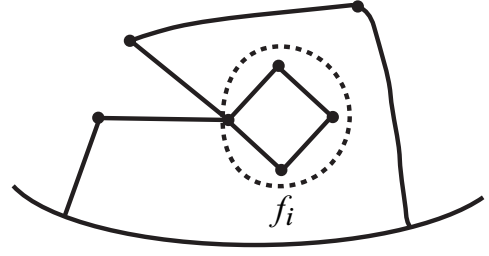

Figure 46

Similarly, if two vertices of $f_{i}$ correspond to the same vertex, $v$, of $\Gamma(\mathcal{T})$, there would be a circle intersecting $\Gamma(\mathcal{T})$ only in $v$ (Figure 46). This would give rise to a crossing that could be reduced by an isotopy rel $\partial B^{3}$.

Now assume $v_{j}$ is incident to $f_{i}$ with $j \neq i, i+1$ (Figure 47). Then $e_{j} \notin f_{i}$ for otherwise $\mathcal{T}$ would be split. Thus $e_{j}$ lies in the exterior of $f_{i}$. But now we can untwist



Figure 47



Figure 48

the crossing at $v_{j}$ by bringing $e_{j}$ into $f_{i}$ (Figure 48).

Theorem 4.7 If $\mathcal{T}$ is a 3-string tangle which can be freely isotoped to a projection with at most seven crossings, then either $\mathcal{T}$ is split or has two parallel strands. 
Proof Note that $\mathcal{T}$ being split or having parallel strands is invariant under free isotopy. Freely isotop $\mathcal{T}$ to a minimal projection with $\leq 7$ crossings and let $\Gamma(\mathcal{T})$ be the corresponding graph. Assume $\mathcal{T}$ is not split. By Lemma 4.4, $\Gamma(\mathcal{T})$ has at least 6 vertices (thus $\mathcal{T}$ has at least 6 crossings). If the six $v_{i}$ are the only vertices incident to $\bigcup_{i} f_{i}$, then (Lemma 4.6) we must have Figure 49.



Figure 49



Figure 50

But then $\mathcal{T}$ has a closed curve, a contradiction.

Thus $\Gamma(\mathcal{T})$ must have another vertex $v$ lying on $f_{1}$, say, which is not a $v_{i}$. Since $\mathcal{T}$ has at most 7 crossings, there is exactly one such $v$ and we have Figure 50. Enumerating the possibilities, we see there are nine cases, six of which are shown in Figure 51, while the remaining three can be obtained from the top three via reflection:
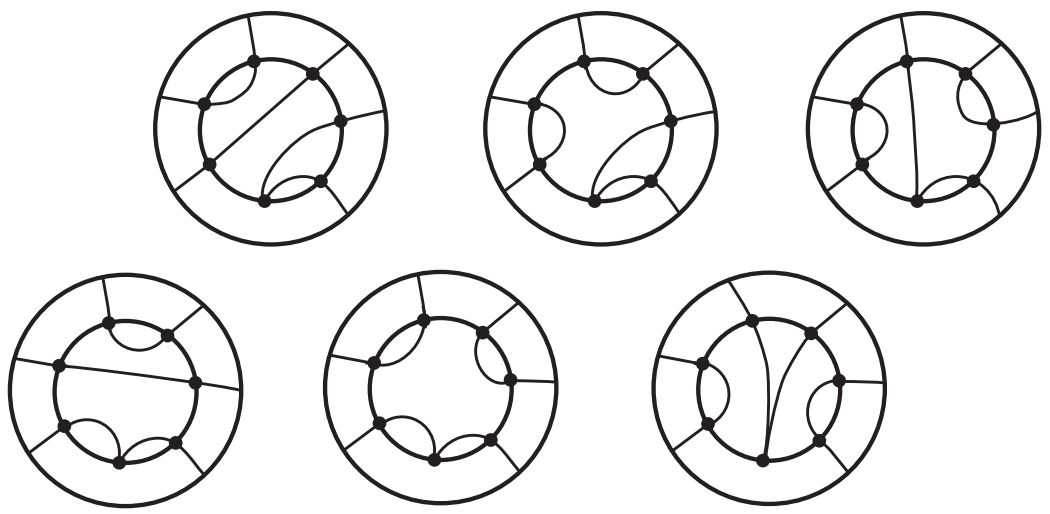

Figure 51

Note in each case we see two parallel strands (since we assumed $\mathcal{T}$ is not split). For example, see Figure 52. 




Figure 52

Theorem 4.7 and Corollary 3.32 imply the following:

Corollary 4.8 Assume $\mathcal{T}$ is a solution tangle. If $\mathcal{T}$ can be freely isotoped to a projection with at most seven crossings, then $\mathcal{T}$ is the PJH tangle.

\section{If $\mathcal{T}$ is not the $\mathrm{PJH}$ tangle, then $\mathcal{T}$ has at least 10 crossings}

Because the three DNA segments captured by the Mu transpososome in [38] are relatively short (50, 175 and 190 base pairs), one can argue that any projection of the transpososome must have few crossings. Let $\mathcal{T}$ be an in trans solution tangle - a tangle satisfying both the in cis and the in trans deletion experiments. In this section we show that if $\mathcal{T}$ is not the PJH tangle (Figure 13), then $\mathcal{T}$ must be complicated, as measured by its minimal crossing number:

Proposition 5.1 Let $\mathcal{T}$ be an in trans solution tangle. If $\mathcal{T}$ has a projection with fewer than 10 crossings, then $\mathcal{T}$ is the PJH tangle.

In this section, we are interested in crossing number up to tangle equivalence, that is, up to isotopy of the tangle fixed on the boundary. So we must fix a framing for our in trans solution tangle. We take that of Proposition 2.5, the normal form.

Remark As pointed out in Section 2, one goes from an in trans solution tangle in normal framing to one in the framing of [38] by adding one left-handed twist at $c_{2}$. Thus, it formally follows from Proposition 5.1 that if an in trans solution tangle under the PJH framing has fewer than 9 crossings, it must be the solution found in [38] (Figure 1). 
The proof of Proposition 5.1 breaks down into checking cases of possible tangle diagrams. We focus on the fact that $s_{12} \cup s_{13}=\mathcal{T}-s_{23}$ is the $-1 / 2$ tangle. Lemma 5.2 shows that each pair of strings must cross at least twice. Hence if an in trans solution tangle has less than 10 crossings, $s_{12} \cup s_{13}$ contains at most 5 crossings. Theorems 5.5, 5.8, 5.13 and 5.15 handle the cases when $s_{12} \cup s_{13}=2,3,4,5$, respectively. In each case it is shown that an in trans solution tangle with less than 10 crossings can be isotoped to a tangle with 7 crossings. By Corollary 4.8, such a tangle is the PJH tangle. This section holds more generally under normal framing when the in trans deletion product is the $(2,2)$ torus link while the remaining deletion products are $\left(2, L_{i}\right)$ torus links where $L_{i} \geq 4$.

Lemma 5.2 Let $\mathcal{T}$ be a solution tangle. In any projection of $\mathcal{T},\left|s_{i j} \cap s_{i k}\right| \geq 2$.

Proof Assume not. Then $\left|s_{i j} \cap s_{i k}\right|=0$. But then $\ell k\left(s_{i j} \cup x_{i j}, s_{i k} \cup x_{i k}\right)=0$, contradicting Lemma 2.3.

Let $\mathcal{T}$ be an in trans solution tangle with crossing number less than 10 . We assume that $s_{23}$ is the enhancer strand. To simplify notation we set $e=s_{23}, \alpha_{2}=s_{12}, \alpha_{3}=s_{13}$. Recall that $\mathcal{T}-e=\alpha_{2} \cup \alpha_{3}$ is the $-1 / 2$-tangle.

Assumption 5.3 $\mathcal{T}$ has a projection, in normal form (see the beginning of Section 2), with at most nine crossings. Furthermore, among all such projections, take $\alpha_{2} \cup \alpha_{3}$ to have the fewest crossings.

Remark We will often blur the distinction between $e, \alpha_{2}, \alpha_{3}$ and their projections.

We will first prove in Theorem 5.5 that Proposition 5.1 holds when $\alpha_{2} \cup \alpha_{3}$ has exactly two crossings. In this case let $R_{1}, R_{2}, R_{3}$ be the closures of the complementary regions of $\alpha_{2} \cup \alpha_{3}$ in Figure 53. Note all figures in this section have been rotated by 90



Figure 53

degrees, and thus the tangle $\mathcal{T}-e=-1 / 2$ is displayed as an integral 2 tangle. 
Lemma 5.4 If $e$ crosses $R_{1} \cap\left(\alpha_{2} \cup \alpha_{3}\right)$ fewer than four times then $\mathcal{T}$ can be freely isotoped to eliminate two crossings. Furthermore if $e \cap R_{1}$ contains only four crossings of $\mathcal{T}$ then we can reduce $\mathcal{T}$ by two crossings unless $e \cap R_{1}$ is as pictured in Figure 54 (up to symmetry).

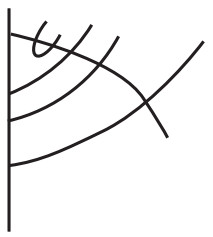

(a)

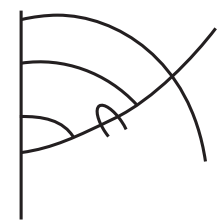

(b)

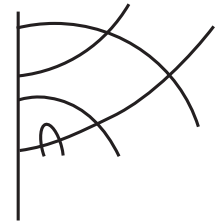

(c)

Figure 54

Proof Assume $e$ intersects $R_{1} \cap\left(\alpha_{2} \cup \alpha_{3}\right)$ in fewer than four crossings. By Lemma 5.2, $e$ must cross $R_{1} \cap\left(\alpha_{2} \cup \alpha_{3}\right)$ exactly twice. Then $R_{1}$ writes $\mathcal{T} \cup c_{1}$ as a disk sum. As $\mathcal{T} \cup c_{1}$ is a rational 2 -string tangle, $e \cap R_{1}$ must be an integral summand [35; 20]. But then we can reduce the crossing number by at least two under a free isotopy by removing the crossings in the integral tangle $e \cap R_{1}$ as well as the two crossings where $e$ intersects $R_{1} \cap\left(\alpha_{2} \cup \alpha_{3}\right)$. See Figure 55. Thus we assume $e \cap R_{1}$ must have at
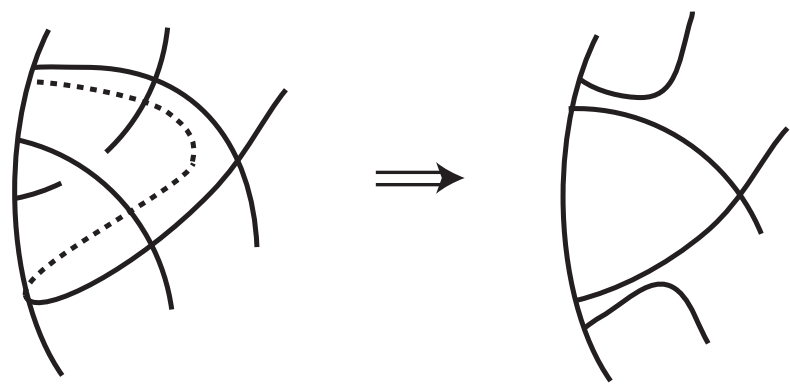

Figure 55

least 4 crossings in $R_{1}$ with $\alpha_{2} \cup \alpha_{3}$. Assume these are the only crossings of $\mathcal{T}$ in $R_{1}$. Then there are no crossings in int $R_{1}$. This allows for two crossing reductions except in the cases pictured in Figure 54. See Figure 56.

Theorem 5.5 Let $\mathcal{T}$ be an in trans solution tangle satisfying Assumption 5.3. Under this projection, assume $\alpha_{2} \cup \alpha_{3}$ has exactly two crossings. Then $\mathcal{T}$ can be freely isotoped to have at most seven crossings. 


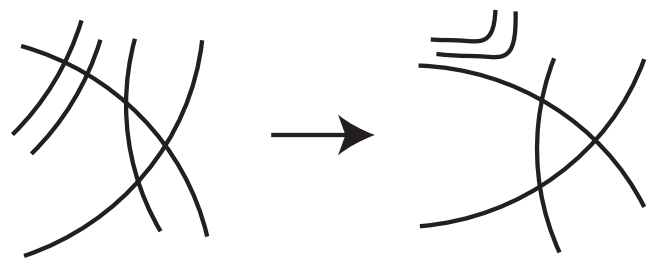

Figure 56

Proof Let $R_{1}, R_{2}, R_{3}$ be the closures of the complementary regions of $\alpha_{2} \cup \alpha_{3}$ in Figure 53.

We divide the proof of Theorem 5.5 into two cases.

Case I $e$ has 4 intersections in $R_{1}$.

Case II $e$ has at least 5 intersections in $R_{1}$.

Proof of Case I $R_{1}$ contains exactly 4 crossings (involving $e$ ). In this case, there are 3 possible configurations up to symmetry (shown in Figure 54) that do not immediately allow a crossing reduction by two in $R_{1}$.

Since there are at most 9 crossings, if $e$ intersects $R_{2}$ then $e \cap R_{2}$ must contribute exactly 2 crossings and $e \cap R_{3}$ must be empty. But this allows a reduction of two crossings: In case (a) and (b) in Figure 54, the two crossing involving $\alpha_{2}$ and $\alpha_{3}$ can be removed as shown in Figure 57. In case (c) one reduction comes from $\alpha_{1} \cap \alpha_{2}$, the

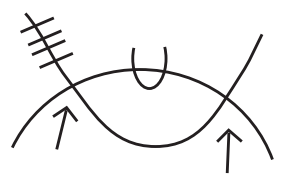

Figure 57

other from $e \cap\left(\alpha_{2} \cup \alpha_{3}\right)$ (Figure 58).

Thus we take $e$ disjoint from $R_{2}$, and we must have (a) or (b) in Figure 59. But (a) gives two crossing reductions and (b) contradicts Lemma 5.2.

Proof of Case II Because $e$ must pick up at least two crossings in $R_{2} \cup R_{3}$, (else we can reduce by two crossings), $e$ must have exactly 4 crossings with $\left(\alpha_{2} \cup \alpha_{3}\right) \cap R_{1}$ and exactly 1 self-crossing inside $R_{1}$. This accounts for nine crossings. For Subcases 1 and 2, we assume the two crossings are with $R_{3}$ - if not, a similar argument works 


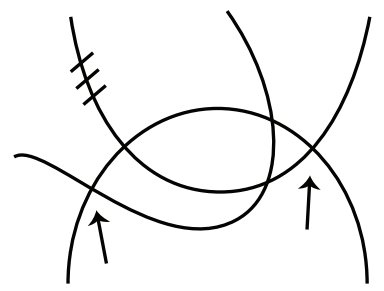

Figure 58

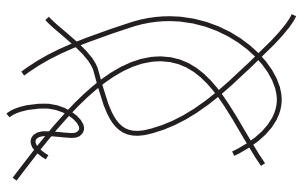

(a)



(b)

Figure 59

if the two crossings are with $R_{2}$ by using the fact that the crossing at $R_{3}$ would be reducible.

Let $\delta_{2}, \delta_{3}$ (in $\alpha_{2}, \alpha_{3}$, resp.) be the arc components of $\left(\alpha_{2} \cup \alpha_{3}-\left(\alpha_{2} \cap \alpha_{3}\right)\right) \cap R_{1}$.

Subcase $1\left|\delta_{2} \cap e\right|=2=\left|\delta_{3} \cap e\right|$ (intersection here refers to the projection).

Then we may assume we have the situation in Figure 60 . Since int $R_{1}$ has exactly one



Figure 60

crossing, the possible cases are shown in Figure 61. In each case we can reduce two crossings.

Subcase $2\left|\delta_{2} \cap e\right|=3,\left|\delta_{3} \cap e\right|=1$ (case when $\left|\delta_{2} \cap e\right|=1,\left|\delta_{3} \cap e\right|=3$ is similar). We have two possibilities, shown in Figure 62.

Recall that $R_{1}$ can have only one more crossing in its interior. If this crossing can be reduced, we can reduce two more crossings by Case 1 . Hence, we assume that this 

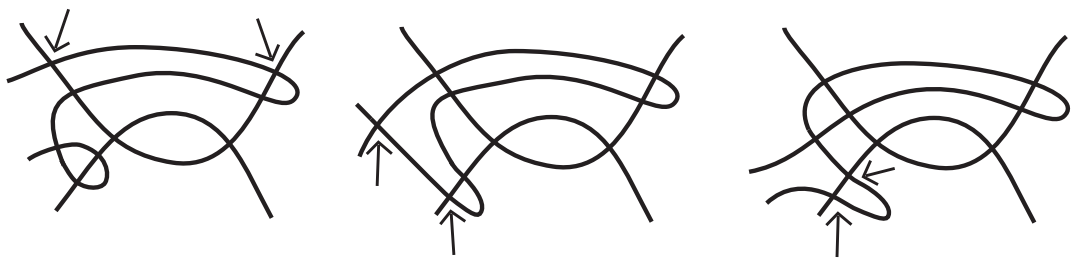

Figure 61

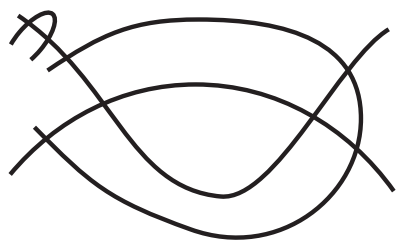

(a)

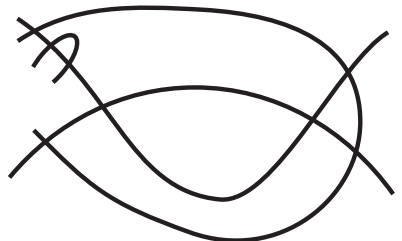

(b)

Figure 62

crossing cannot be reduced. Thus we see that both of the cases in Figure 62 allow a reduction of two crossings, as in Figure 63.

(a)



(b)

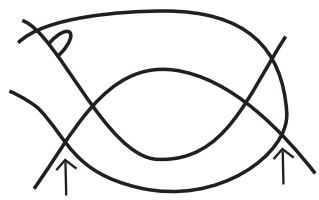

or

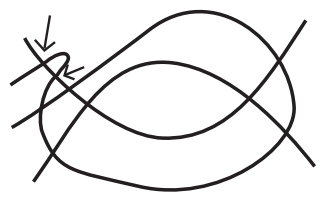

or



Figure 63

Subcase $3\left|\delta_{2} \cap e\right|=4,\left|\delta_{3} \cap e\right|=0$ (case when $\left|\delta_{2} \cap e\right|=0,\left|\delta_{3} \cap e\right|=4$ is similar). Recall $\left|e \cap\left(R_{2} \cup R_{3}\right)\right|=2$. If $\left|e \cap R_{3}\right|=2$, then $\left|e \cap \alpha_{3}\right|<2$, contradicting Lemma 5.2. If $\left|e \cap R_{2}\right|=2$, then we can remove the two crossings of $\alpha_{2} \cap \alpha_{3}$.

Subcases (1)-(3) exhaust all possibilities, showing a reduction in crossing number by two in Case II.

This finishes the proof of Theorem 5.5. 
Definition 5.6 $\alpha_{i}$ has a trivial self-intersection with respect to $\alpha_{j}$ if $\alpha_{i}$ has a selfintersection such that the subarc of $\alpha_{i}$ connecting the double points is disjoint from $\alpha_{j},\{i, j\}=\{2,3\}$.

Lemma 5.7 If $\mathcal{T}$ is an in trans solution tangle satisfying Assumption 5.3 and $\alpha_{i}$ has a trivial self-intersection with respect to $\alpha_{j}$ for $\{i, j\}=\{2,3\}$, then we can reduce $\mathcal{T}$ by two crossings.

Proof Assume $\alpha_{2}$ has a trivial self-intersection with respect to $\alpha_{3}$, and let $\delta \subset \alpha_{2}$ be the subarc connecting its double points. As $\left|\alpha_{2} \cap \alpha_{3}\right|$ is minimal, Figure 64 shows that we may assume $|\delta \cap e| \geq 4$.


Figure 64

By Lemma 5.2, $\left|\alpha_{2} \cap \alpha_{3}\right| \geq 2$ and $\left|e \cap \alpha_{3}\right| \geq 2$. Thus $|\delta \cap e|=4,\left|\alpha_{2} \cap \alpha_{3}\right|=2$, $\left|e \cap \alpha_{3}\right|=2$ and $\alpha_{2}$ has a self crossing, accounting for all nine crossings. Let $\alpha_{2}^{\prime}=\alpha_{2}-\operatorname{int}(\delta)$. Let $R_{1}, R_{2}, R_{3}$ be the closures of the complementary regions of $\alpha_{2}^{\prime} \cup \alpha_{3}$ as in Figure 53. Then $\left|e \cap \partial R_{1}\right| \geq 2$ and $\left|e \cap\left(\partial R_{2} \cup \partial R_{3}\right)\right| \geq 2$, otherwise we can reduce by two the number of crossings. Thus $\left|e \cap\left(\alpha_{2} \cup \alpha_{3}\right)\right| \geq 8$. Because $\left|\alpha_{2} \cap \alpha_{3}\right| \geq 2$, we have too many crossings.

Theorem 5.8 Let $\mathcal{T}$ be an in trans solution tangle satisfying Assumption 5.3. If $\alpha_{2} \cup \alpha_{3}$ contributes three crossings to $\mathcal{T}$ (including self-crossings), then $\mathcal{T}$ can be freely isotoped to have at most seven crossings.

Proof Let $\mathcal{T}$ be such an in trans solution tangle. Then $\alpha_{3}$, say, must have a selfintersection which we may assume is not trivial with respect to $\alpha_{2}$. Thus $\alpha_{2} \cup \alpha_{3}$ must be as in Figure 65, with $R_{1}, R_{2}$ complementary components of $\alpha_{2} \cup \alpha_{3}, R_{1}$ containing the endpoints of $e$, and $\delta_{1}, \delta_{2}, \ldots, \delta_{8}$ the arc components of $\alpha_{2} \cup \alpha_{3}-\left(\alpha_{2} \cap \alpha_{3}\right)$.

Claim 5.9 If $\left|e \cap\left(R_{1} \cap\left(\alpha_{2} \cup \alpha_{3}\right)\right)\right|<4$, then $\mathcal{T}$ can be reduced by two crossings.

Proof We may assume that $\left|e \cap\left(R_{1} \cap\left(\alpha_{2} \cup \alpha_{3}\right)\right)\right|=2$. Then a copy of $\partial R_{1}$ writes $\mathcal{T} \cup c_{1}$ as a disk sum. Since $\mathcal{T} \cup c_{1}$ is a rational tangle, one of the summands must be integral with respect to to the disk. This must be the left-hand side, $R_{1} \cap e$. After a 


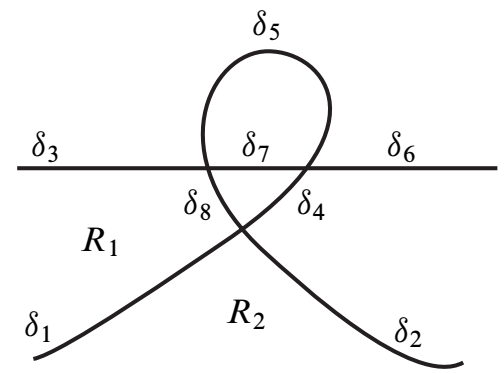

Figure 65

free isotopy we may take this to be $0 / 1$. Hence we can take $e$ to have no self-crossings in $R_{1}$.

Let $\delta_{1}, \delta_{2}$ be as pictured in Figure 65. If $\left|e \cap \delta_{1}\right|=2$, then we can freely isotop away two crossings. If $\left|e \cap \delta_{1}\right|=1$, then $\left|e \cap \delta_{2}\right| \geq 3$ (else we can reduce two crossings in $R_{2}$ ). Since $\left|e \cap \alpha_{2}\right| \geq 2$ by Lemma 5.2, $\left|e \cap \alpha_{2}\right|$ must be two, accounting for all intersections. That is, if $\left|e \cap \delta_{1}\right|=1$ we are as in Figure 66, where we can reduce by two crossings. Thus we assume $e$ is disjoint from $\delta_{1}$.

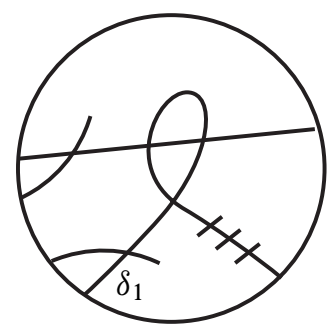

Figure 66

Similarly we show that $e$ is disjoint from $\delta_{3}$ (see Figure 65). If $\left|e \cap \delta_{3}\right|=2$, then we can reduce by two crossings in $R_{1}$. So assume $\left|e \cap \delta_{3}\right|=1$. If $e \cap \delta_{2}$ is empty we can reduce two crossings (the self-intersection and $e \cap \delta_{3}$ ), so $\left|e \cap \delta_{2}\right| \geq 2$. The only possibility is shown now in Figure 67, which we can reduce by two crossings.

So we assume $e$ is disjoint from $\operatorname{arcs} \delta_{1}$ and $\delta_{3}$ in $\partial R_{1}$. See Figure 68.

Now $\left|e \cap \alpha_{2}\right| \geq 2$ by Lemma 5.2, and $\left|e \cap \delta_{2}\right| \geq 2$ (else we can reduce two crossings). This accounts for all nine crossings. But we must also have a crossing between $e$ and $\delta_{4} \cup \delta_{5}$.

Claim 5.10 e must intersect $\delta_{1}$. 


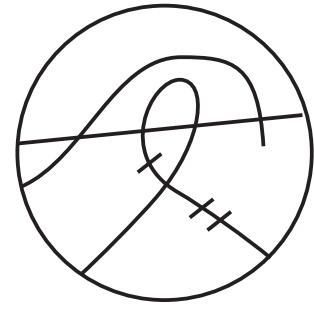

Figure 67

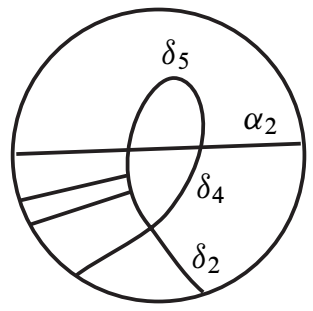

Figure 68

Proof By Claim 5.9, we may assume $\left|e \cap\left(R_{1} \cap\left(\alpha_{1} \cup \alpha_{3}\right)\right)\right| \geq 4$. Label the arc components of $\alpha_{2} \cup \alpha_{3}-\left(\alpha_{2} \cap \alpha_{3}\right)$ as in Figure 65. Assume $e$ is disjoint from $\delta_{1}$. It cannot also be disjoint from $\delta_{2}$, otherwise we can eliminate the crossing at $\delta_{1} \cap \delta_{2}$ and argue as in Case I of Theorem 5.5. Thus $e$ must intersect $\delta_{2}$ exactly twice, thereby accounting for all crossings. But the crossings of $e$ at $\delta_{2}$ lead to additional crossings.

Claim 5.11 $\mathcal{T}$ can be reduced by two crossings if $\left|e \cap\left(R_{1} \cap\left(\alpha_{2} \cup \alpha_{3}\right)\right)\right|<6$.

Proof By Claim 5.9, we may assume $\left|e \cap\left(R_{1} \cap\left(\alpha_{1} \cup \alpha_{3}\right)\right)\right|=4$.

Subclaim $5.12 e \cap\left(\delta_{6} \cup \delta_{7}\right)$ is nonempty.

Proof Suppose $e \cap\left(\delta_{6} \cup \delta_{7}\right)$ is empty. Then $\left|e \cap \delta_{3}\right|=2$ by Lemma 5.2. If $e$ crosses $\delta_{5}$ then it must cross twice, accounting for all crossings of $\mathcal{T}$. Then there are no crossings in int $R_{1}$. Since $\left|e \cap \delta_{1}\right|>0$ and $\left|e \cap \delta_{3}\right|=2$, we have two crossing reductions in $R_{1}$. Thus $e$ is disjoint from $\delta_{5}$.

Similarly $e$ must not cross $\delta_{2}$. If $\left|e \cap \delta_{2}\right| \neq 0$, then there can be no crossings in int $R_{1}$. Since $\left|e \cap \delta_{1}\right|>0$ and $\left|e \cap \delta_{3}\right|=2$, we see the crossing reductions for $\mathcal{T}$. Since $e$ does not cross $\delta_{2}$, but does cross $\delta_{1}$, e must cross $\delta_{1}$ twice. Hence $e$ also does not $\operatorname{cross} \delta_{8}$.

Thus we assume $e$ does not cross $\delta_{2}, \delta_{5}, \delta_{6}, \delta_{7}$, or $\delta_{8}$. As the 2 -string tangle $\alpha_{2}, \alpha_{3}$ forms a two crossing tangle, we see that the crossings between $\alpha_{2}$ and $\alpha_{3}$ may be reduced in $\mathcal{T}$.

Assume first that $e$ crosses $\delta_{7}$. Then $e$ must cross $\delta_{5} \cup \delta_{7}$ twice, accounting for all crossings. Then $e$ does not cross $\delta_{2}$ and crosses $\delta_{1}$ exactly two times. These crossings can be reduced.

Thus $e$ crosses $\delta_{6}$. If $e$ does not cross $\delta_{2}$ then we again see two crossings at $\delta_{1}$ that can be reduced. If $e$ crosses $\delta_{2}$ then it crosses it once, accounting for all crossings. 
Thus $e$ crosses each of $\delta_{1}$ and $\delta_{3}$ an odd number of times and has no self-crossings in int $R_{1}$. Enumerating the possibilities one sees that we can reduce the crossings of $e$ at $\delta_{3}, \delta_{6}$ or at $\delta_{1}, \delta_{2}$.

This finishes the proof of Claim 5.11.

By Claim 5.11 we may assume $\left|e \cap R_{1} \cap\left(\alpha_{2} \cup \alpha_{3}\right)\right|=6$. This accounts for all crossings of $\mathcal{T}$. By Lemma 5.2, $\left|e \cap \delta_{3}\right| \geq 2$. As all crossings are accounted for, two of the crossings of $e$ with $\delta_{1} \cup \delta_{3}$ can be eliminated by a free isotopy.

Thus we have proved Theorem 5.8.

Theorem 5.13 Let $\mathcal{T}$ be an in trans solution tangle satisfying Assumption 5.3. If $\alpha_{2} \cup \alpha_{3}$ has four crossings (including self-crossings), then there is a free isotopy of $\mathcal{T}$ reducing it to at most seven crossings.

Proof Assume $\mathcal{T}$ is as hypothesized. By Lemma 5.7, $\alpha_{2} \cup \alpha_{3}$ has no trivial loops and we enumerate the possibilities for $\alpha_{2} \cup \alpha_{3}$ in Figure 69 where $R_{1}$ is the closure of the (planar) complementary region of $\alpha_{1} \cup \alpha_{2}$ containing the ends of $e$ and where $\delta_{1}, \delta_{2}$ are the extremal arc components of $\alpha_{2} \cup \alpha_{3}-\left(\alpha_{2} \cap \alpha_{3}\right)$ in $R_{1}$.

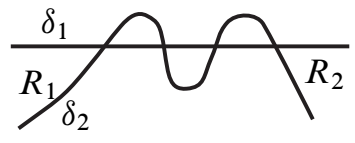

(a)



(b)

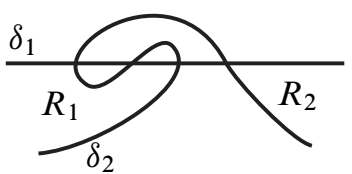

(c)

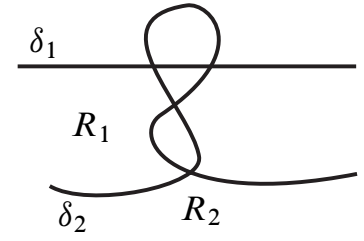

(d)



(e)

Figure 69

Lemma 5.14 With $\mathcal{T}$ as in the hypothesis of Theorem 5.13, if e contains a selfcrossing then that self-crossing appears in $R_{1}$. 
Proof Assume a self-crossing appears in a complementary region $R \neq R_{1}$ of $\alpha_{2} \cup \alpha_{3}$. Since $\left|\alpha_{2} \cap \alpha_{3}\right|=4,\left|e \cap \alpha_{2}\right|=2\left|e \cap \alpha_{3}\right|=2$, e can have at most one self-crossing. Hence $R$ has exactly 5 crossings involving $e$ : four from $e$ crossing $\alpha_{2} \cup \alpha_{3}$ and one self-crossing. By Lemma 5.2, e must cross $\alpha_{2}$ twice and $\alpha_{3}$ twice in $R$. Exactly one pair of these crossings must be in a component of $\alpha_{2} \cup \alpha_{3}-\left(\alpha_{2} \cap \alpha_{3}\right)$ shared with $R_{1}$ (all crossings are accounted for). If this component is $\delta_{1}$ or $\delta_{2}$ we may reduce by two crossings. So we assume this pair is in a different component. Looking at the possibilities of Figure 69, we immediately rule out (a) and (b). In cases (c), (d) and (e), we can reduce two crossings of $\alpha_{2} \cup \alpha_{3}$ since we have accounted for all crossings (for case (d), note $e$ must cross each of $\alpha_{2}$ and $\alpha_{3}$ twice in $R$ ).

We continue the proof of Theorem 5.13.

Case I $e$ crosses $R_{1} \cap\left(\alpha_{2} \cup \alpha_{3}\right)$ exactly twice.

If both crossings are in $\delta_{1} \cup \delta_{2}$, then we can reduce by two crossings. This rules out configurations (a),(b) of Figure 69. If $\mathcal{T}$ has nine crossings, then one must be a self-crossing. By Lemma 5.14 it must occur in $R_{1}$ and hence can be untwisted to reduce the crossing number by one. Thus we assume $\mathcal{T}$ has only 8 crossings. Hence $e$ must be disjoint from $\delta_{1} \cup \delta_{2}$. In cases (c), (d), and (e), $e$ must then be disjoint from the region labelled $R_{2}$. But then we can reduce the crossing at $R_{2}$, thus completing Case I.

Case II $e$ crosses $R_{1} \cap\left(\alpha_{2} \cup \alpha_{3}\right)$ four times.

If $\mathcal{T}$ has nine crossings then one must be a self-crossing of $e$. By Lemma 5.14, the self-crossing occurs in $R_{1}$. Thus, whether $\mathcal{T}$ has eight or nine crossings, all crossings of $\mathcal{T}$ involving $e$ lie in $R_{1}$. By Lemma 5.2, two of the crossings of $e$ are with $\alpha_{2}$ and two with $\alpha_{3}$. Looking at Figure 69, we see that $e$ must have exactly two crossings with $\delta_{i}$ for $i=1$ or 2 (in cases (c) and (e) we could otherwise reduce by two crossings). Either we can reduce by two crossings or near $\delta_{i}$ we have Figure 70. This writes $\mathcal{T} \cup c_{1}$

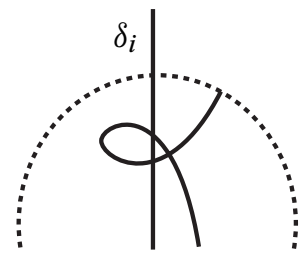

Figure 70

as a disk sum. As $\mathcal{T} \cup c_{1}$ is integral with respect to the disk slope, each summand is 
integral with respect to to the disk slope. But this means we can eliminate the crossings at $\delta_{i}$ by a free isotopy of $\mathcal{T}$. This completes the proof of Case II and hence of Theorem 5.13 .

Theorem 5.15 Let $\mathcal{T}$ be an in trans solution tangle satisfying Assumption 5.3. If $\alpha_{2} \cup \alpha_{3}$ has five crossings (including self-crossings), then there is a free isotopy of $\mathcal{T}$ reducing it to at most seven crossings.

Proof Let $\mathcal{T}$ be such a tangle. Without loss of generality assume $\alpha_{2}$ has at most one self-intersection. By Lemma $5.2 e$ must cross each of $\alpha_{2}, \alpha_{3}$ exactly twice. Since $e$ can contribute at most 4 crossings this accounts for all crossings coming from $e$. In particular, $e$ has no self-crossings.

Case I $\alpha_{2}$ crosses $\alpha_{3}$ twice.

We have three possibilities as shown in Figure 71 (Lemma 5.7), where $R_{1}$ is the complementary region of $\alpha_{2} \cup \alpha_{3}$ containing the ends of $e$ ( $\ell$ is discussed below). In case (c) we may reduce the crossings of $\mathcal{T}$ by two $(\mathcal{T}$ has no local knots, hence

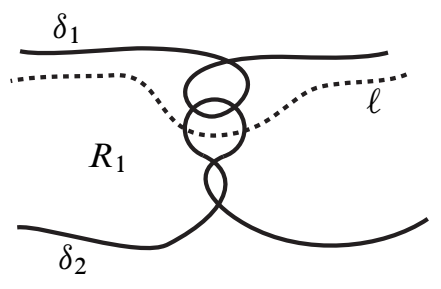

(a)

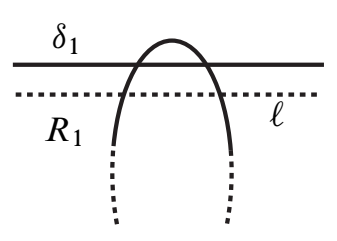

(b)

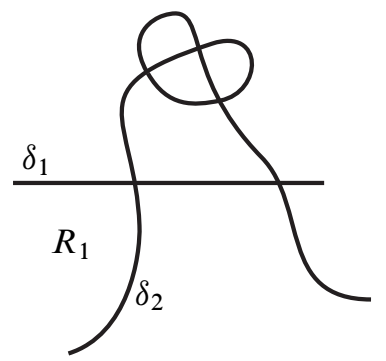

(c)

Figure 71

$\left.\left|e \cap\left(\delta_{1} \cup \delta_{2}\right)\right|=2\right)$. So we restrict our attention to (a) and (b) and consider the arc $\ell$ in Figure 71. Since $\left|e \cap \alpha_{2}\right|=2$, $\ell$ intersects $e$ twice. Hence $e \cup \ell$ is as in Figure 72, where $e^{\prime}, e^{\prime \prime}$ are components of $e-\ell$. Let $\ell^{\prime}$ be the arc pictured in Figure 72, consisting of $e^{\prime \prime}$ and part of $\ell$.

\section{Claim 5.16 $\left|\ell^{\prime} \cap \alpha_{3}\right|=2$.}

Proof $\left|\ell^{\prime} \cap \alpha_{3}\right|$ is even and at least 2 , since the endpoints of $\alpha_{3}$ are below $\ell^{\prime}$. If $\left|\ell^{\prime} \cap \alpha_{3}\right| \geq 4$, then $\left|e^{\prime \prime} \cap \alpha_{3}\right|=2=\left|\left(\ell^{\prime}-e^{\prime \prime}\right) \cap \alpha_{3}\right|$. Going back to Figure 71 we see we can eliminate the crossings of $e$ and $\alpha_{2}$. 


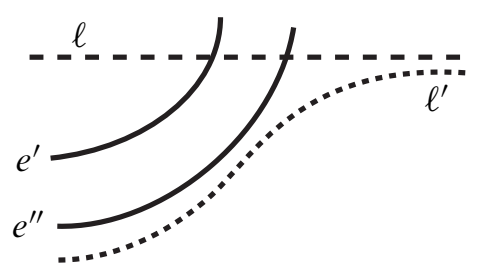

Figure 72

By Claim 5.16, $\ell^{\prime}$ writes $\mathcal{T} \cup c_{2}$ as a disk sum. By Lemma 2.1, if $\mathcal{T}$ is a normal form solution tangle, $\mathcal{T} \cup c_{2}$ is the $-1 / 4$ tangle. As the crossing number of the summand below $\ell^{\prime}$ is at most 3 , the tangle below $\ell^{\prime}$ is integral with respect to the disk slope allowing us to eliminate the crossings there. Thus there is at most one crossing below $\ell^{\prime}$. Now we can eliminate two crossings from $\mathcal{T}$, where $\alpha_{3}$ crosses $e^{\prime \prime}$ or $\alpha_{2}$ near where $\alpha_{3}$ crosses $\ell^{\prime}$. So we have finished Case I.

Case II $\alpha_{2}$ crosses $\alpha_{3}$ four times.

Without loss of generality assume $\alpha_{2}$ has no self-intersections and $\alpha_{3}$ only one. Then $\alpha_{2} \cup \alpha_{3}$ must be one of the cases in Figure 73 .

Claim 5.17 $\left|e \cap\left(\delta_{1} \cup \delta_{2}\right)\right| \leq 1$.

Proof Assume $\left|e \cap\left(\delta_{1} \cup \delta_{2}\right)\right| \geq 2$. If $\left|e \cap R_{1} \cap\left(\alpha_{2} \cup \alpha_{3}\right)\right| \leq 2$, then the crossings at $\delta_{1} \cup \delta_{2}$ can be eliminated. So assume $\left|e \cap R_{1} \cap\left(\alpha_{2} \cup \alpha_{3}\right)\right|=4$, accounting for all crossings of $e$. Then $\left|e \cap \delta_{i}\right|=2$ for some $i=1,2$, and we can reduce these crossings.

Claim 5.17 eliminates cases (1a), (2a), (3a), (4a). If $\left|e \cap \delta_{i}\right|=1$ then it must give rise to a reducible crossing in $R_{1}$. In cases $(2 \mathrm{~b}),(3 \mathrm{~b}),(4 \mathrm{~b})$ we could then reduce also the crossing at $R_{2}$. In these cases then we assume $e$ disjoint from $\delta_{1} \cup \delta_{2}$. By inspection we now see we can reduce by two crossings $\left(\left|e \cap\left(\alpha_{2} \cup \alpha_{3}\right)\right|=4\right)$.

We are left with (1b), (5a) and (5b). If $\left|e \cap \delta_{2}\right|=1$, then we can eliminate two crossings at $e \cap R_{2}$. If $\left|e \cap \delta_{1}\right|=1$, then we can eliminate this crossing and the self-crossing of $\alpha_{3}$. With $\left|e \cap \alpha_{2}\right|=2=\left|e \cap \alpha_{3}\right|$ and $e \cap\left(\delta_{1} \cup \delta_{2}\right)$ empty, we see that in all possibilities we can eliminate two crossings from $\mathcal{T}$.

Thus we have finished the proof of Theorem 5.15. 


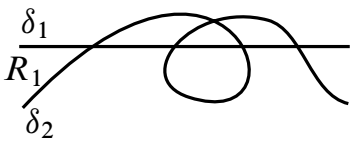

(1a)

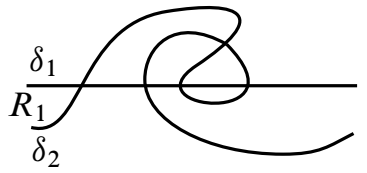

(2a)

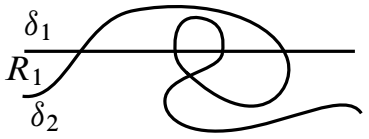

(3a)

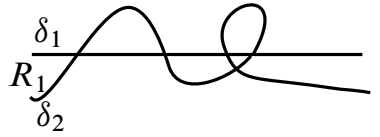

(4a)



(5a)

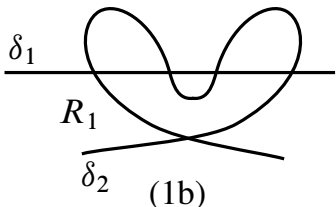

(1b)

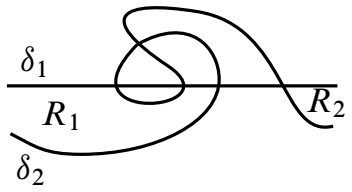

(2b)

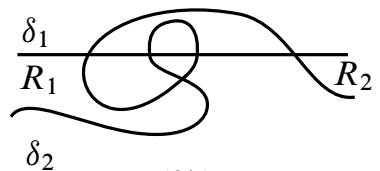

(3b)

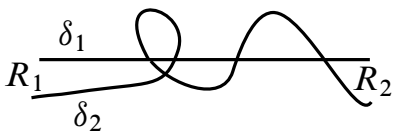

(4b)

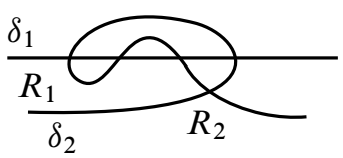

(5b)

Figure 73

\section{References}

[1] C Alén, D Sherratt, S Colloms, Direct interaction of aminopeptidase A with recombination site DNA in Xer site-specific recombination, EMBO J. 16 (1997) 5188-5197

[2] J Arsuaga, Y Diao, M Vazquez, Mathematical methods in DNA topology: Applications to chromosome organization and site-specific recombination, from: "Mathematics of DNA Structure, Function, and Interactions", (C J Benham, S Harvey, W K Olson, D W Sumners, editors), IMA Vol. Math. Appl. 150, Springer, New York (2009) 7-36

[3] T Baker, K Mizuuchi, DNA-promoted assembly of the active tetramer of the Mu transposase, Genes Dev. 6 (1992) 2221-2232

[4] A Bates, A Maxwell, DNA Topology, second edition, Oxford Univ. Press (2005)

[5] W Bauer, F Crick, J White, Supercoiled DNA, Sci. Am. 243 (1980) 100-113 
[6] S A Bleiler, RA Litherland, Lens spaces and Dehn surgery, Proc. Amer. Math. Soc. 107 (1989) 1127-1131 MR984783

[7] D Buck, C Verjovsky Marcotte, Tangle solutions for a family of DNA-rearranging proteins, Math. Proc. Cambridge Philos. Soc. 139 (2005) 59-80 MR2155505

[8] H Cabrera-Ibarra, On the classification of rational 3-tangles, J. Knot Theory Ramifications 12 (2003) 921-946 MR2017963

[9] H Cabrera-Ibarra, Results on the classification of rational 3-tangles, J. Knot Theory Ramifications 13 (2004) 175-192 MR2047467

[10] C Calladine, H Drew, B Luisi, A Travers, Understanding DNA, third edition, Academic Press, London (2004)

[11] A J Casson, C M Gordon, Reducing Heegaard splittings, Topology Appl. 27 (1987) 275-283 MR918537

[12] G Chaconas, R Harshey, Transposition of phage Mu DNA, from: "In Mobile DNA II”, (H Craig, R Craigie, M Gellert, A Lambowitz, editors), Amer. Soc. Microbiology (2002) 384-402

[13] S Colloms, J Bath, D Sherrat, Topological selectivity in Xer site-specific recombination, Cell 88 (1997) 855-864

[14] I K Darcy, Biological distances on DNA knots and links: applications to XER recombination, from: "Knots in Hellas '98, Vol. 2 (Delphi)", J. Knot Theory Ramifications 10 (2001) 269-294 MR1822492

[15] I K Darcy, Modeling protein-DNA complexes with tangles, Comput. Math. Appl. 55 (2008) 924-937 MR2389822

[16] I K Darcy, J Chang, N Druivenga, C McKinney, R Medikonduri, S Mills, J Navarra-Madsen, A Ponnusamy, J Sweet, T Thompson, Coloring the Mu transpososome, BMC Bioinformatics 7 (2006) 435

[17] I K Darcy, R G Scharein, TopoICE-R: 3D visualization modelling the topology of DNA recombination, Bioinformatics 22 (2006) 1790-1791

[18] P L Deininger, J V Moran, M A Batzer, H H Kazazian, Jr, Mobile elements and mammalian genome evolution, Curr. Opin. Genet. Dev. 13 (2003) 651-658

[19] J Emert, C Ernst, N-string tangles, J. Knot Theory Ramifications 9 (2000) 987-1004 MR1807603

[20] C Ernst, D W Sumners, A calculus for rational tangles: applications to DNA recombination, Math. Proc. Cambridge Philos. Soc. 108 (1990) 489-515 MR1068451

[21] C Ernst, D W Sumners, Solving tangle equations arising in a DNA recombination model, Math. Proc. Cambridge Philos. Soc. 126 (1999) 23-36 MR1681651

[22] C M Gordon, On primitive sets of loops in the boundary of a handlebody, Topology Appl. 27 (1987) 285-299 MR918538 
[23] I Grainge, M Bregu, M Vasquez, V Sibanathan, S Ip, D Sherrat, Unlinking chromosome catenates in vivo by site-specific recombination, EMBO J. 26 (2007) 4228-4238

[24] I Grainge, D Buck, M Jayaram, Geometry of site alignment during Int family recombination: antiparallel synapsis by the Flp recombinase, J. Mol. Biol. 298 (2000) 749-764

[25] F Guo, D Gopaul, G van Duyne, Structure of Cre recombinase complexed with DNA in a site-specific recombination synapse, Nature 389 (1997) 40-46

[26] R Harshey, M Jayaram, The Mu transpososome through a topological lens, Crit. Rev. Biochem. Mol. Biol. 41 (2006) 387-405

[27] M Hirasawa, K Shimokawa, Dehn surgeries on strongly invertible knots which yield lens spaces, Proc. Amer. Math. Soc. 128 (2000) 3445-3451 MR1676336

[28] H Hoess, M Ziese, N Sternberg, P1 site-specific recombination: Nucleotide sequence of the recombining sites (dyad symmetry/lox site/BAL-31 deletion/plasmid recombination), Proc. Natl. Acad. Sci. USA 79 (1982) 3398-3402

[29] W Jaco, Adding a 2-handle to a 3-manifold: an application to property $R$, Proc. Amer. Math. Soc. 92 (1984) 288-292 MR754723

[30] M Jayaram, R Harshey, Difference topology: Analysis of high-order DNA-protein assemblies, from: "Mathematics of DNA Structure, Function, and Interactions", (C J Benham, S Harvey, W K Olson, DW Sumners, D Swigon, editors), IMA Vol. Math. Appl. 150, Springer, New York (2009) 139-158

[31] E Kilbride, M Boocock, W Stark, Topological selectivity of a hybrid site-specific recombination system with elements from Tn3 res/resolvase and bacteriophase PL lox P/Cre, J. Mol. Biol. 289 (1999) 1219-1320

[32] S Kim, K Darcy, Isabel, Topological analysis of DNA-protein complexes, from: "Mathematics of DNA Structure, Function, and Interactions", (C J Benham, S Harvey, W K Olson, D W Sumners, D Swigon, editors), IMA Vol. Math. Appl. 150, Springer, New York (2009) 177-194

[33] P Kronheimer, T Mrowka, P Ozsváth, Z Szabó, Monopoles and lens space surgeries, Ann. of Math. (2) 165 (2007) 457-546 MR2299739

[34] W Li, S Kamtekar, Y Xiong, G Sarkis, N Grindley, T Steitz, Structure of a synaptic $\gamma \delta$ resolvase tetramer covalently linked to two cleaved DNAs, Science 209 (2005) 1210-1215

[35] W B R Lickorish, Prime knots and tangles, Trans. Amer. Math. Soc. 267 (1981) 321332 MR621991

[36] M Miziuuchi, T Baker, K Mizuuchi, Assembly of the active form of the transposaseMu DNA complex: a critical control point in Mu transposition, Cell 70 (1992) 303-311

[37] K Mouw, S Rowland, M Gajjar, M Boocock, W Stark, P Rice, Architecture of a serine recombinase-DNA regulatory complex, Molecular Cell 30 (2008) 145-155 
[38] S Pathania, M Jayaram, R Harshey, Path of DNA within the Mu Transpososome: Transposase interaction bridging two Mu ends and the enhancer trap five five DNA supercoils, Cell 109 (2002) 425-436

[39] S Pathania, M Jayaram, R Harshey, A unique right end-enhancer complex precedes synapsis of Mu ends: the enhancer is sequestered within the transpososome throughout transposition, EMBO J. 22 (2003) 3725-3736

[40] N Robertson, P D Seymour, R Thomas, Linkless embeddings of graphs in 3-space, Bull. Amer. Math. Soc. (N.S.) 28 (1993) 84-89 MR1164063

[41] S Rowland, W Stark, M Boocock, Sin recombinase from Staphylococcus aureus: synaptic complex architecture and transposon targeting, Mol. Microbiol. 44 (2002) 607-619

[42] Y Saka, M Vazquez, Topological analysis of site-specific recombination, Bioinformatics 18 (2002) 1011-1012

[43] D Sankoff, Rearrangements and chromosomal evolution, Curr. Opin. Genet. Dev. 13 (2003) 583-587

[44] M Scharlemann, Outermost forks and a theorem of Jaco, from: "Combinatorial methods in topology and algebraic geometry (Rochester, N.Y., 1982)”, (J R Harper, R Richard Mandelbaum, editors), Contemp. Math. 44, Amer. Math. Soc. (1985) 189-193 MR813113

[45] M Scharlemann, A Thompson, Detecting unknotted graphs in 3-space, J. Differential Geom. 34 (1991) 539-560 MR1131443

[46] W Stark, D Sherratt, M Boocock, Site-specific recombination by Tn3 resolvase: topological changes in the forward and reverse reactions, Cell 58 (1989) 779-790

[47] D W Sumners, Lifting the curtain: using topology to probe the hidden action of enzymes, Notices Amer. Math. Soc. 42 (1995) 528-537 MR1324733

[48] D W Sumners, C Ernst, N Cozzarelli, S Spengler, Mathematical analysis of the mechanisms of DNA recombination using tangles, Quarterly Reviews of Biophysics 28 (1995) 253-313

[49] A Thompson, A polynomial invariant of graphs in 3-manifolds, Topology 31 (1992) 657-665 MR1174264

[50] M Vazquez, S Colloms, D W Sumners, Tangle analysis of Xer recombination reveals only three solutions, all consistent with a single three-dimensional topological pathway, J. Mol. Biol. 346 (2005) 493-504

[51] M Vazquez, D W Sumners, Tangle analysis of Gin site-specific recombination, Math. Proc. Cambridge Philos. Soc. 136 (2004) 565-582 MR2055047

[52] A Vetcher, A Lushnikov, J Navarra-Madsen, R Scharein, Y Lyubchenko, I K Darcy, S Levene, DNA topology and geometry in Flp and Cre recombination, J. Mol. Biol. 357 (2006) 1089-1104 
[53] C Wang, Helical repeat of DNA in Solution, Proc. Natl. Acad. Sci. USA 76 (1979) 200-208

[54] S Wasserman, J Dungan, N Cozzarelli, Discovery of a predicted DNA knot substantiates a model for site-specific recombination, Science 229 (1985) 171-174

[55] J Watson, F Crick, The structure of DNA, Cold Spring Harb. Symp. Quant. Biol. 18 (1953) 123-131

[56] Y Q Wu, A generalization of the handle addition theorem, Proc. Amer. Math. Soc. 114 (1992) 237-242 MR1070535

[57] Y Q Wu, On planarity of graphs in 3-manifolds, Comment. Math. Helv. 67 (1992) 635-647 MR1185812

[58] Y-Q Wu, The classification of nonsimple algebraic tangles, Math. Ann. 304 (1996) 457-480 MR1375620

[59] Z Yin, M Jayaram, S Pathania, R Harshey, The Mu transposase interwraps distant DNA sites within a functional transposome in the absence of DNA supercoiling, J. Biol. Chem. 280 (2005) 6149-6156

[60] Z Yin, A Suzuki, Z Lou, M Jayaram, Interactions of phage Mu enhancer and termini that specify the assembly of a topologically unique interwrapped transpososome, J. Mol. Biol. 372 (2007) 382-396

[61] W Zheng, C Galloy, B Hallet, M Vasquez, The tangle model for site-specific recombination: a computer interface and the TnpI-IRS recombination system, from: "Knot Theory for Scientific Objects (Proc. of Intl. Workshop, Osaka, 2006)”, (K Kawauchi, editor), Ocami Studies 1, Osaka Municipal Univ. Press (2007) 251-271

Department of Mathematics, University of Iowa Iowa City, IA 52242, USA

Department of Mathematics, The University of Texas at Austin, 1 University Station C1200, Austin, TX 78712-0257, USA

Department of Mathematics, San Francisco State University

1600 Holloway Avenue, San Francisco, CA 94132, USA

idarcy@math.uiowa.edu, luecke@math.utexas.edu, mariel@math.sfsu.edu http://math.uiowa.edu/ idarcy, http://math.sfsu.edu/mariel/

Received: 31 October 2007 Revised: 14 July 2009 